\title{
Parametric study on the three-dimensional distribution of velocity of a FENE-CR fluid flow through a curved channel
}

\author{
Joana M. Malheiro ${ }^{\mathrm{a}, *}$, Paulo J. Oliveira ${ }^{\mathrm{b}}$, Fernando T. Pinho ${ }^{\mathrm{c}}$ \\ ${ }^{a}$ UMTP - Unidade de Materiais Têxteis e Papeleiros, DEM, Universidade da Beira Interior, 6201-001 Covilhã, Portugal \\ ${ }^{\mathrm{b}}$ Departamento de Engenharia Electromecânica, Universidade da Beira Interior, Research Unit: CEFT, Porto, 6201-001 Covilhã, Portugal

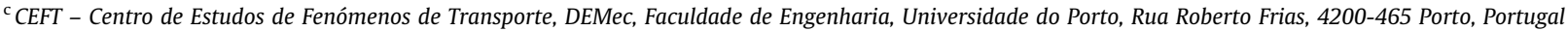

\section{A R T I C L E I N F O}

\section{Article history:}

Received 31 July 2012

Received in revised form 19 December 2012

Accepted 20 December 2012

Available online 27 December 2012

\section{Keywords:}

Curved duct

FENE-CR model

Finite-volume method

Secondary flow

Elasticity effects

\begin{abstract}
A B S T R A C T
In order to better understand the three-dimensional non-Newtonian flow in an $180^{\circ}$ curved duct of square cross-section, simulations were carried out considering an incompressible viscoelastic fluid, which follows the non-linear FENE-CR model, having constant shear viscosity. A fully implicit finite-volume method was used for the solution of the governing equations. Numerical simulations were performed for different Reynolds and Weissenberg numbers, and by varying the model parameters, namely the retardation ratio $(\beta)$ and the extensibility $\left(L^{2}\right)$. The aim was to analyse the development and distribution of velocity field in the cross-sections along the curved channel and as a consequence to understand the variation of maximum velocity with these parameters. The results reveal complex changes with increasing extensibility and decreasing retardation parameter, which are associated to transition from one to two pairs of vortices in the secondary flow. Comparison with the literature confirms and reveals that the absence of shear-thinning delays this transition.
\end{abstract}

(c) 2013 Elsevier B.V. All rights reserved.

\section{Introduction}

The flow of Newtonian and non-Newtonian fluids in curved ducts has important applications in many industries and in medicine $[1,2]$. Despite the fact that there are many studies published on this topic, the mechanism for the onset and development of secondary flows in curved ducts is far from completely understood, especially when the fluids are viscoelastic [1,2]. The interest on flows in curved channels comes from the centrifugal induced secondary flows, first reported by Eustice in 1911 [3] and later theoretically analysed by Dean [4], whose name became associated with curvature effects. The effects of secondary flow can be beneficial, as in the improvement of heat and mass transfer or in the increase of cross-section mixing and residence time of fluid particles, among others [1,2], or inconvenient for the same purpose since the flow tends to be more stable in what regards transition to turbulence in comparison to the flow in a straight duct $[1,5]$.

The secondary flow in curved ducts is induced by imbalanced forces associated with centrifugal effects that create a radial pressure gradient leading to flow from the inner wall to the outer wall regions through the central part of the channel cross-section and balanced by a return flow from the outer wall to the inner wall

* Corresponding author. Address: Calçada Fonte do Lameiro - Universidade da Beira Interior, Departamento Engenharia Electromecânica, 6201-001 Covilhã, Portugal. Tel.: +351275329952.

E-mail address: joanamouramalheiro@gmail.com (J.M. Malheiro). along the top and bottom channel walls. In the laminar regime the secondary flow in a curved channel with square cross-section is characterised by a pair of symmetrical vortices that occupy the entire cross-section of the channel. The magnitude of this secondary flow is characterised by the Dean number $(\mathrm{Dn})$ which is generally defined as the ratio of inertial or centrifugal forces to viscous forces, and given by an expression of the type [6]:

$D n=\frac{R e}{\sqrt{R_{c}}}$

where $R e$ is the Reynolds number, $R_{c}$ is the dimensionless curvature ratio (here $R_{c}=R_{m} / a$, and $R_{m}$ is the actual mean radius of curvature and $a$ is the side of the square cross-section of the channel). However, in some conditions (e.g. higher Reynolds number) the secondary flow becomes more complex, with the appearance of an additional pair of counter rotating vortices near the outer wall and possible flow unsteadiness. For Newtonian fluids the transition from one to two pairs of vortices is described with some detail by Bara et al. [6].

The same type of phenomenon was already observed for nonNewtonian viscoelastic fluids although in this case as a consequence of more complex and less understood effects. Fellouah et al. [7] numerically and experimentally studied Dean instabilities using power-law and Bingham fluids in a curved duct of rectangular cross-section. Independently, Iemoto et al. [8] and Das [9] verified that the secondary flow of a purely viscous fluid (following a 
power law [8] and a Bingham [9] model) is weaker than that of a viscoelastic fluid (considering a White-Metzner model), in a curved pipe. From a similarity solution for an Oldroyd-B fluid flowing in the region between two circular concentric cylinders of large radius, with radial injection of fluid at the outer cylinder wall, Phan-Thien and Zheng [10] found that the flow kinematics was very close to that for a Newtonian fluid under the same conditions. In this problem, secondary flows were absent. More relevant to the present work, in a series of studies of flows in annular spaces with rectangular cross-section, Joo and Shaqfeh [11-13] reported a purely elastic flow instability, which had not been studied before and was found to be stationary in pressure driven Dean flow of an Oldroyd-B fluid. In addition, they showed that this so-called purely elastic Dean flow is destabilized by inertia. For the same fluid model, Sarin [14] studied the effects of Deborah number on the position of the maximum axial velocity and of the centre of vortices, and verified an increase in the magnitude of secondary flows, in curved pipe. The effects of rotation about $z$-axis (normal to the flow direction axis) on secondary flow intensity, axial velocity and axial normal stress were investigated by Zahng et al. [15] for an Oldroyd-B fluid flow in a curved duct of square cross-section. They concluded that all the parameters they focused in are affected by rotation, generating multiple pairs of vortices. Recently, Norouzi et al. [16] focused on the effects of centrifugal force due to the curvature of the rectangular cross-section duct and the opposite effects of the first and second normal stress differences on the flow field, considering a second-order fluid. Their numerical results showed that while the first normal stress difference favours the transition from one to two pairs of vortices, the second normal stress difference has the opposite effect. Also considering a second-order fluid, but flowing in a curved pipe, Sharma and Prakash [17] established that the first normal stress difference intensifies the secondary flow. Also for a curved pipe, Fan et al. [18] subsequently confirmed the finding for the Oldroyd-3-constant fluid model (which includes the Upper-Convective-Maxwell and Oldroyd-B models) and showed that the negative second normal stress
Table 1

Reynolds and Dean number equivalents for the geometry considered.

\begin{tabular}{llll}
\hline$\sqrt{R_{m} / a}=\sqrt{15.1}$ & & & \\
\hline$R e$ & 486 & 532 & 583 \\
$D n$ & 125 & 137 & 150 \\
\hline
\end{tabular}

Table 2

Mesh characteristics.

\begin{tabular}{llrllll}
\hline & & $N X \times N Y \times N Z$ & $f_{X}$ & \multicolumn{1}{l}{$f_{Y}$} & \multicolumn{1}{l}{$f_{Z}$} & NCV \\
\hline MESH 1 & Block I & $30 \times 20 \times 20$ & 0.95212 & 1.00000 & 1.00000 & 88400 \\
& Block II & $161 \times 20 \times 20$ & 1.00000 & 1.00000 & 1.00000 & \\
& Block III & $30 \times 20 \times 20$ & 1.05029 & 1.00000 & 1.00000 & \\
MESH 2 & Block I & $30 \times 20 \times 20$ & 0.91772 & 1.00000 & 1.00000 & 152400 \\
& Block II & $321 \times 20 \times 20$ & 1.00000 & 1.00000 & 1.00000 & \\
& Block III & $30 \times 20 \times 20$ & 1.08965 & 1.00000 & 1.00000 & \\
MESH 3 & Block I & $30 \times 30 \times 30$ & 0.95118 & 1.00000 & 1.00000 & 198900 \\
& Block II & $161 \times 30 \times 30$ & 1.00000 & 1.00000 & 1.00000 & \\
& Block III & $30 \times 30 \times 30$ & 1.05133 & 1.00000 & 1.00000 & \\
\hline
\end{tabular}

difference has the opposite effect, i.e., it decreases the intensity of the secondary flow. Helin et al. [19] presented results on the development of the flow in a curved duct with square cross-section considering two viscoelastic fluids, namely the Oldroyd-B model and a modified version of the Phan-Thien-Tanner (PTT) model. For the former, the magnitude and intensity of the secondary vortices increase with elasticity, and for the later the onset of the second pair of vortices in the secondary flow takes place at lower Reynolds numbers as Deborah number increases. The later result was also numerically verified by Boutabaa et al. [20], considering the same geometry.

The present work is driven by the need to better understand viscoelastic fluid flow in curved ducts under inertial conditions and in particular the changes in the flow characteristics due to the presence of fluid elasticity. More precisely, the aim is to analyse the

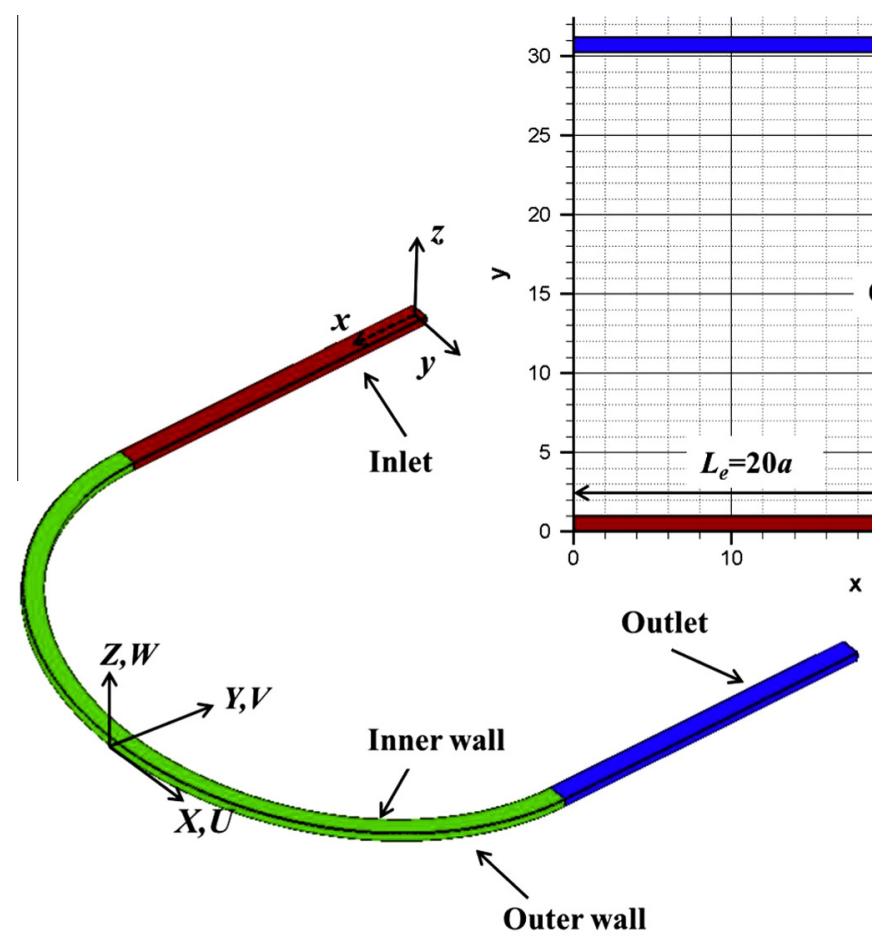

Fig. 1. Geometry of the curved channel. 


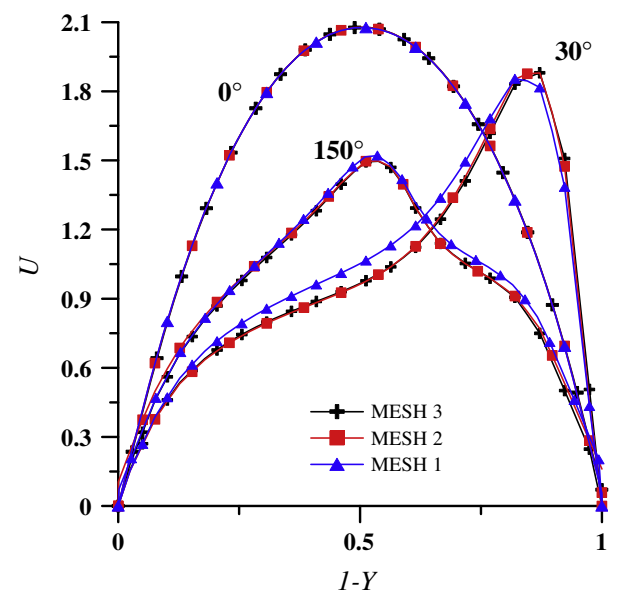

Fig. 2. Mesh refinement comparison for $R e=583, W i=0.5, L^{2}=100$ and $\beta=0.5$.

effects of the extensibility $\left(L^{2}\right)$ and the retardation $(\beta)$ parameters of the constant-viscosity viscoelastic fluid on the development of axial velocity distribution across the section and along the curve, considering the three-dimensional curved channel represented in Fig. 1. The geometry consists of an $180^{\circ}$ curved duct, with internal radius
$R_{1}=14.6 a$ and external radius $R_{2}=15.6 a$, therefore defining a mean radius of curvature of $R_{m}=\frac{1}{2}\left(R_{1}+R_{2}\right)=15.1 a$, coupled to two straight ducts, one at the entrance and the other at the exit, both with lengths $L_{e}=20 a$. All ducts have a square cross-section of width $a=1$. The geometry is the same as those of other authors $[6,7,21]$.

\section{Governing equations and non-dimensional parameters}

In this work, the flow is assumed to be three-dimensional, isothermal, laminar and steady. The fluids are incompressible and are either Newtonian or follow the rheological FENE-CR model (Finitely Extensible Non-linear Elastic formulated by Chilcott-Rallison [22]). The governing equations are the mass conservation and momentum equations:

$\nabla \boldsymbol{u}=0$

$\rho \frac{\partial \boldsymbol{u}}{\partial t}+\rho \boldsymbol{u} \cdot \nabla \boldsymbol{u}=-\nabla p+\nabla \cdot \tau_{\text {tot }}$

where $\boldsymbol{u}$ is the velocity vector, $\nabla$ the gradient operator, $\rho$ the fluid density, $t$ the time, $p$ the pressure and $\tau_{\text {tot }}$ the extra stress tensor. The extra stress tensor is defined as $\tau_{\text {tot }}=\tau_{s}+\tau$, where $\tau_{s}$ is the contribution of the solvent, which follows the Newtonian constitutive equation with viscosity $\eta_{s}$, and $\tau$ is the contribution of the polymer molecules defined by the FENE-CR [22] model:
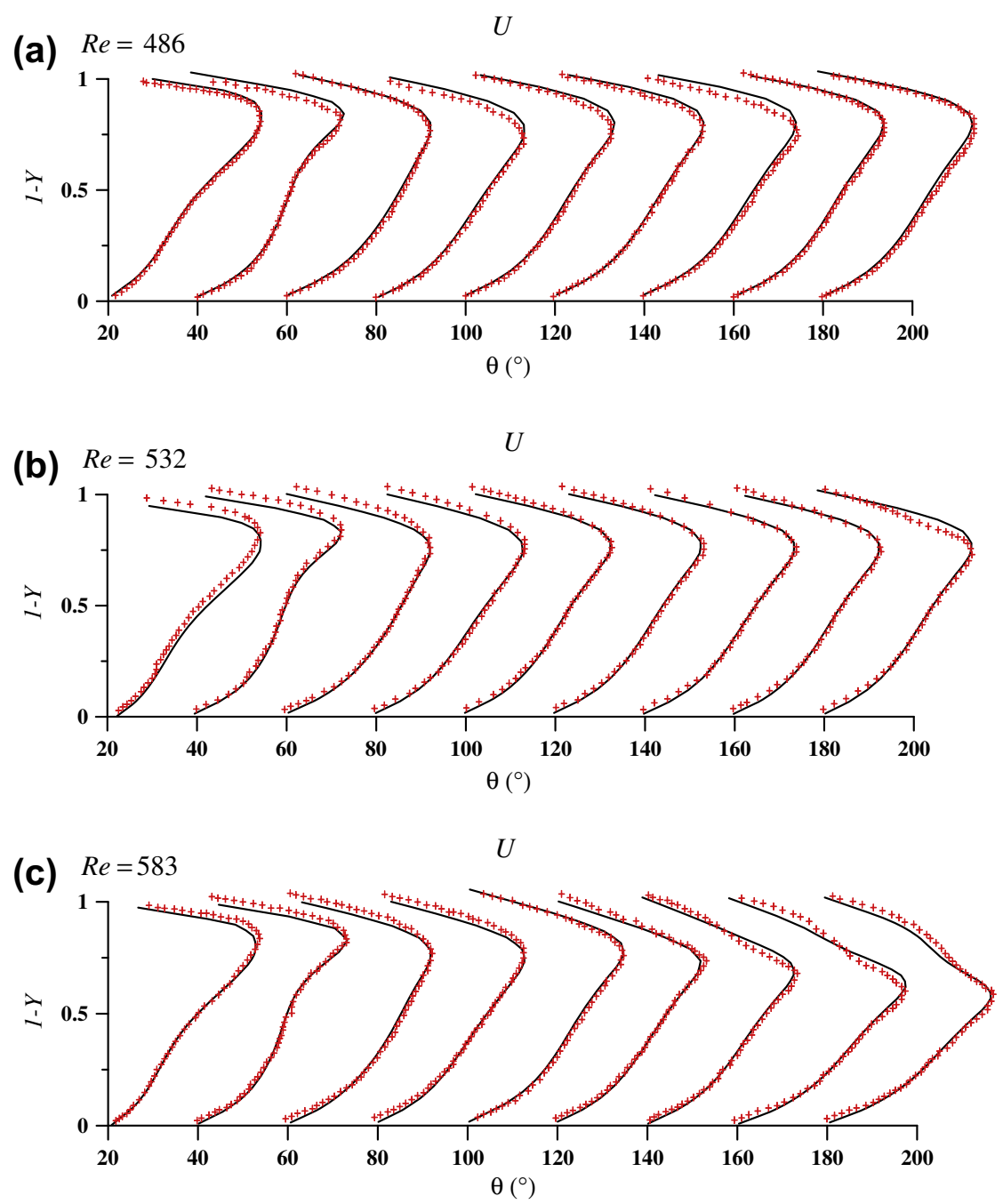

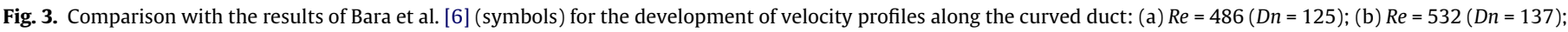
(c) $R e=583(D n=150)$. 
$\tau+\left(\frac{\nabla}{\lambda} \underset{f(\tau)}{\tau}\right)=2 \eta_{p} \boldsymbol{D}$

In this equation, the rate of strain tensor is $\boldsymbol{D}=1 / 2\left(\nabla \boldsymbol{u}+\nabla \boldsymbol{u}^{T}\right)$, $\eta_{p}$ is the polymer viscosity parameter and $\lambda$ is the constant zeroshear rate relaxation time.

The FENE-CR [22] model results from an empirical modification of the original FENE model [23], which is based on the kinetic theory of polymer molecules with finitely and non-linear elastic extension, in order to describe a constant shear viscosity elastic fluid (a Boger fluid having viscosity $\eta=\eta_{s}+\eta_{p}$ ). Therefore, this model allows the study of elastic effects without the influence of shear-thinning in shear viscosity [24]. Nevertheless, the FENE-CR model exhibits shear-thinning in the first-normal stress difference coefficient $\left(\Psi_{1}\right)$, controlled by the extensibility function $f(\tau)$, which is given by:

$f(\tau)=\frac{L^{2}+\left(\frac{\lambda}{\eta_{p}}\right) \operatorname{tr}(\tau)}{L^{2}-3}$

In the model, the maximum molecular stretch is measured by the so-called extensibility parameter $L^{2}$, defining the size of the fully-extended dumbbell representing the polymer molecules in relation to its equilibrium size.

For the flow of a fluid having viscosity $\eta$ in a curved duct of square cross-section with side $a$ and mean averaged velocity $U_{m}$ $\left(U_{m}=Q / a^{2}\right.$, where $Q$ is the flow rate), the relevant dimensionless parameters are the Reynolds number:

$\operatorname{Re}=\frac{\rho U_{m} a}{\eta}$

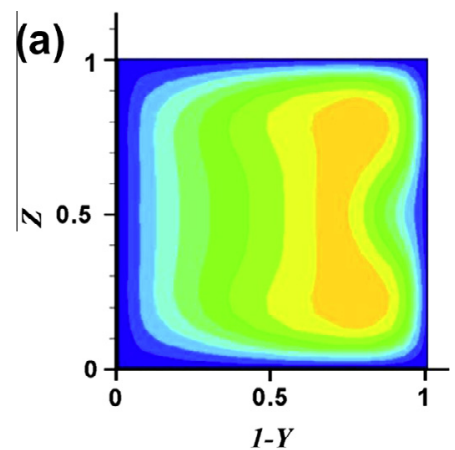

representing the ratio of inertial to viscous forces, the Dean number defined in Eq. (1), which is a modification of the Reynolds number by the path curvature (Table 1 shows the equivalents for the geometry considered), and the Weissenberg number:

$$
W i=\dot{\gamma} \lambda \quad\left(\text { with characteristic shear rate }\left(\dot{\gamma}=U_{m} / a\right)\right)
$$

which measures the influence of the ratio of the elastic normal forces to the polymeric contribution to the viscous shear forces. Although FENE models do not allow a clear separation of the effects of elasticity related to extensional influence (measured by $L^{2}$ ) and the effects of elasticity related to shear-induced normal-stress differences (measured by $\beta$ and $W i$ ) these latter two parameters may be combined. Hence, in addition to $W i$ we have also used a modified Weissenberg number defined as $W i_{\text {mod }}=(1-\beta) W i$ which relates the standard Weissenberg number Wi (Eq. (7)) with the retardation parameter $\beta$, in order to make implicit the elastic influence of $\beta$. The retardation parameter expresses the relation between the retardation time $\left(\lambda_{r}\right)$ and the relaxation time $(\lambda)$, and is defined by: $\beta=\lambda_{r} / \lambda$

\section{Numerical method}

The set of governing Eqs. (2)-(4) was solved numerically by a finite-volume method [25], which has already been described in a number of previous studies [25-27] so the present explanation can be kept concise. The scheme is fully implicit and it is formulated on a general non-orthogonal coordinate system and a collocated mesh arrangement. This method guarantees that mass is conserved and momentum is balanced consistently with other forces, over each individual control volume and over the whole

(b)



Fig. 4. Representation of the planes of data extraction: (a) at the cross-section and (b) at different angular positions in the curve.

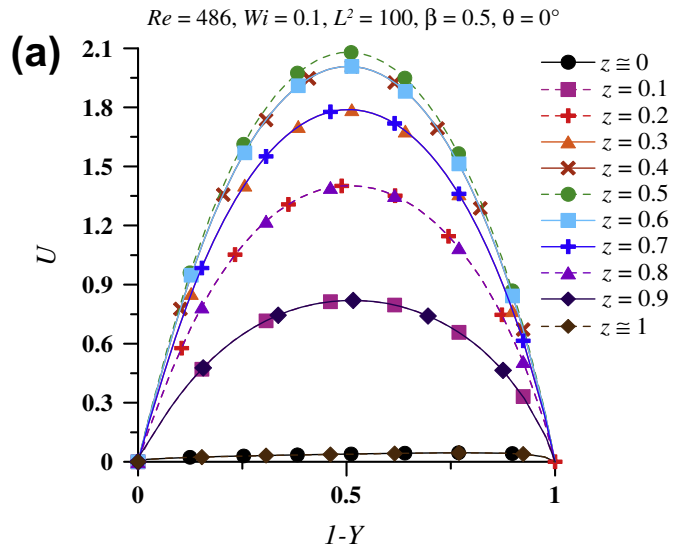

(b)

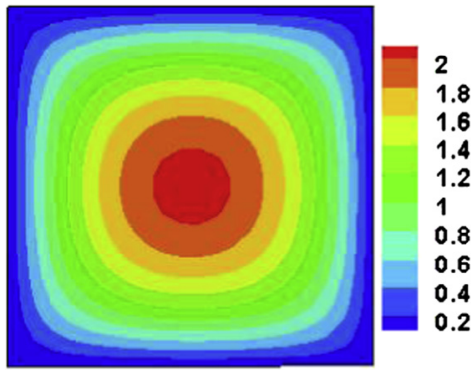

Fig. 5. Fully developed flow at the entrance to the curve: (a) velocity profiles extracted from the cross-section; and (b) 2D axial velocity distribution. 

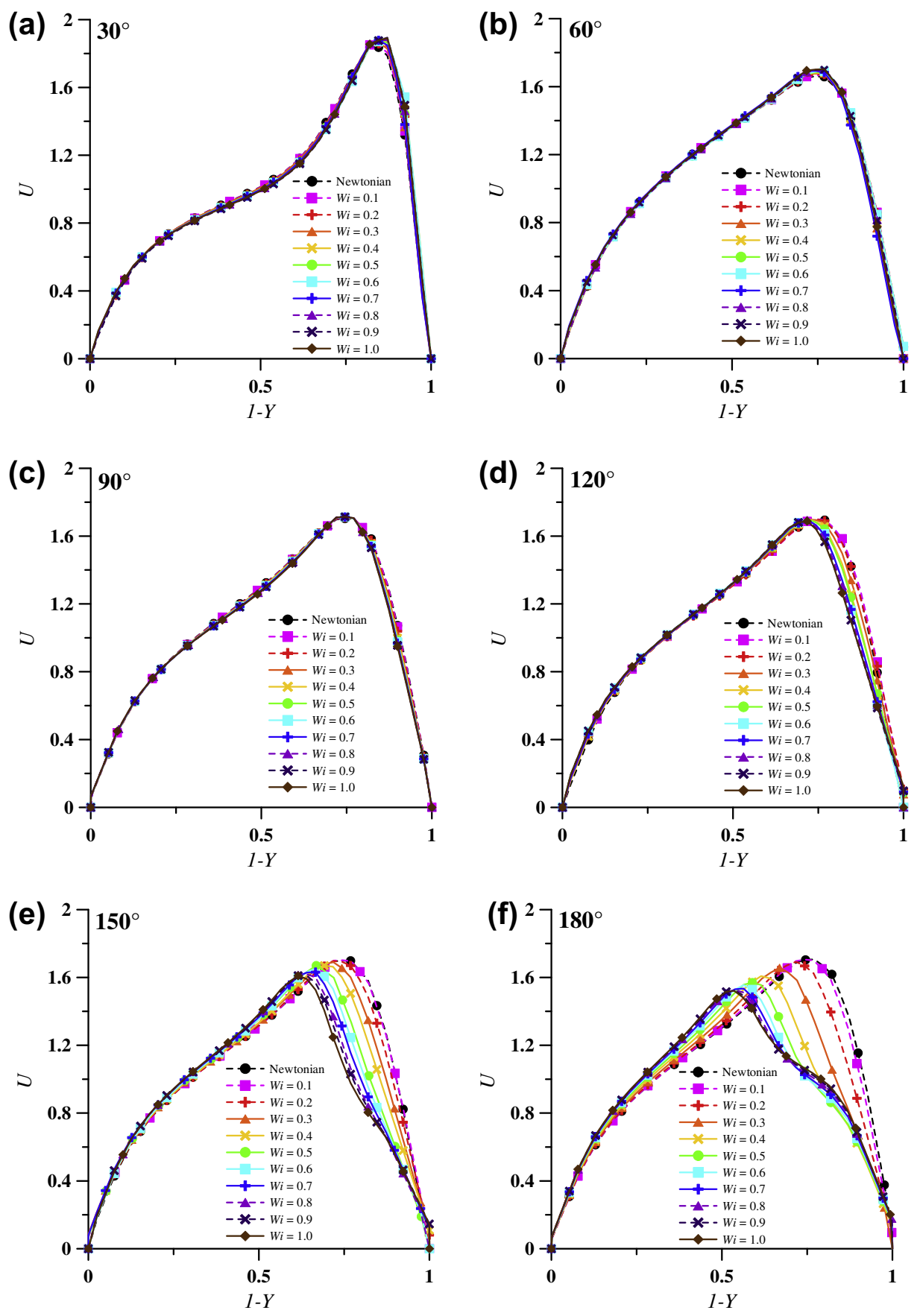

Fig. 6. Variation of axial velocity $U$ with $W i$ along the curve. Velocity profiles extracted at the middle plane $Z=0.5$, for $R e=532, L^{2}=100$ and $\beta=0.5$.

domain, with all variables (velocities, pressure and stresses) calculated at the centre of the control-volumes, which are organised to form a structured computational mesh. The discretization of the governing equations is of second-order. The CUBISTA scheme [26] was applied to approximate the advective terms, the central differences method was applied to the diffusive and source terms, and the three-time level method, explained by Oliveira [27] in the context of viscoelastic simulations, was applied to the unsteady terms (a steady flow solution is not assumed a priori and the final flow may be unsteady). The solution algorithm ensures the coupling of the velocity and pressure fields through an iterative process of correcting pressure and velocity, so that continuity is eventually verified, and was applied as in Oliveira [27], where a complete description of the procedure is given.
The computational mesh was generated by blocks (represented by different colours in Fig. 1) and the mesh is non-uniform along the length of the inlet and outlet straight channels and uniform along the curve and along the cross-section of the whole length of the channel. The mesh used in the simulations was designed after a mesh refinement study to ensure that the results were not affected by mesh fineness. The characteristics of three different meshes are in Table 2, where MESH 3 is the finest mesh, MESH 2 is the mesh used to obtain the results presented here, and MESH 1 is the less refined mesh. In order to analyse the effect of mesh refinement, we carried out a set of simulations for the case $R e=583$, $W i=0.5, L^{2}=100$ and $\beta=0.5$, using the different meshes. Fig. 2 depicts the velocity profiles at different positions in the curve $\left(\theta=0^{\circ}\right.$, $30^{\circ}$ and $150^{\circ}$ ), and shows that the difference between MESH 2 and 

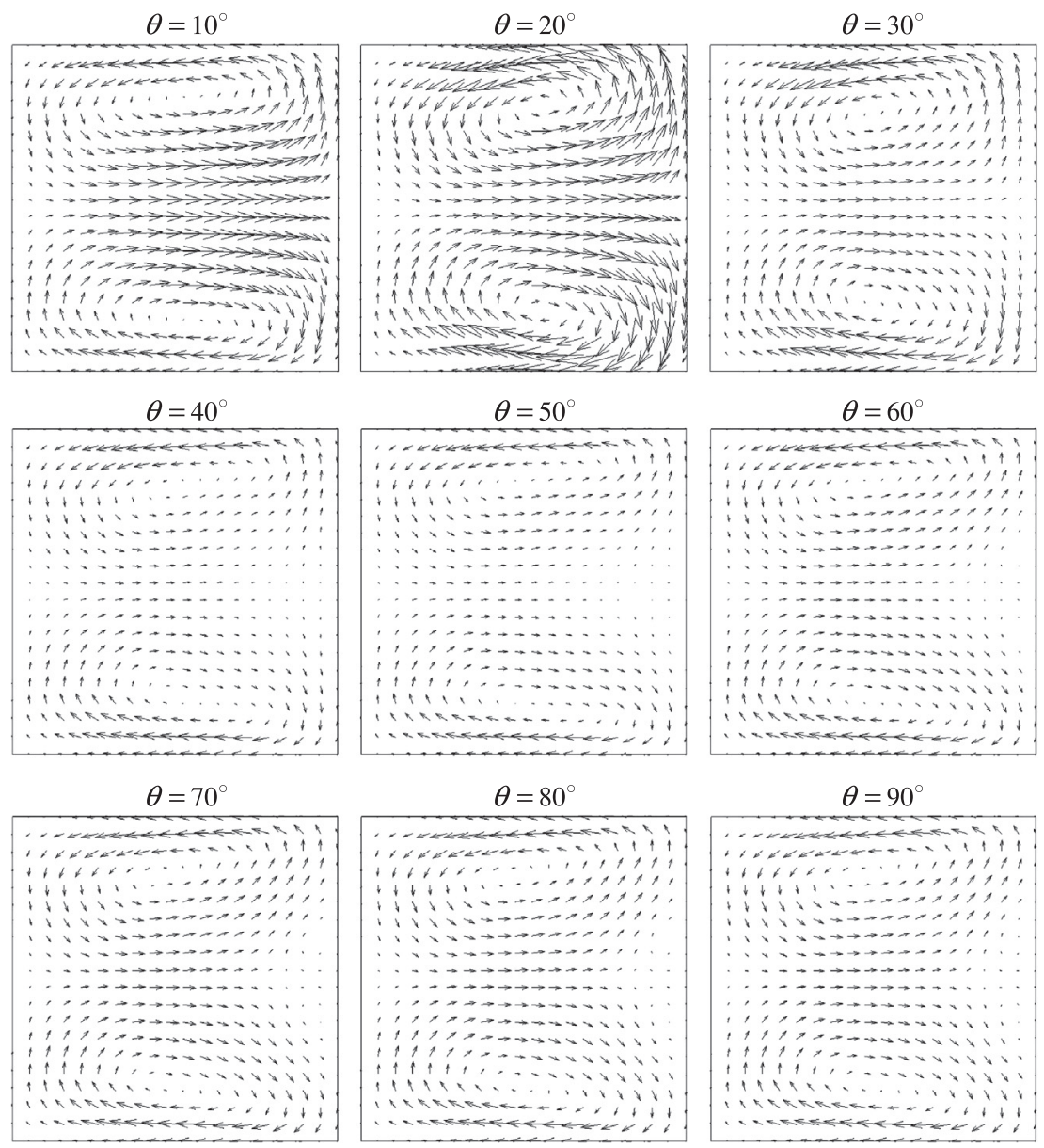

Fig. 7. Secondary flow development in the first half of the curve. Velocity fields for $R e=532, W i=0.5, L^{2}=100$ and $\beta=0.5$.

MESH 3 is hardly noticeable, allowing us to conclude that MESH 2 provides adequate accuracy with less computational time.

No-slip boundary conditions are applied at the walls $(u=v=w=0$ at $Z=Y=0$ and $Z=Y=1$ ). A fully developed velocity profile is imposed at the inlet to the entrance channel $(X=0)$, which is followed by a sufficiently long straight channel ( $L_{e}=20 a$ ) in order to guarantee that the flow is independent of inlet conditions in the curved portion of the channel. For the FENE-CR model, and since its shear viscosity is constant, the fully developed velocity profile is the same of the Newtonian case for which there is a well-known analytical solution. At the exit, a zero streamwisegradient condition was imposed for the velocity and for the pressure gradient. The simulations were carried out using the entire domain of the channel, to account for the possibility of asymmetric flow [28]. The properties of the fluid are considered constant.

\section{Results}

In order to validate the code, we compare our results with the experimental data of Bara et al. [6] in Fig. 3, where velocity profiles extracted from the middle plane of the duct $(Z=0.5)$ at different positions along the curve are presented. The solid lines represent our results and the symbols pertain to the data of Bara et al. [6] for three values of the Reynolds numbers ( $R e=486,532$ and 583). Good agreement was obtained for all cases thus validating the numerical method and confirming again the adequateness of the mesh resolution.

Concerning the flow of the viscoelastic fluids described by the FENE-CR model there are three independent dimensionless numbers that need to be considered, in addition to the Reynolds number and the radius of curvature. These are the flow elasticity (via the Weissenberg number, $W i$ ), the polymer extensibility (via the maximum possible extension $L^{2}$ ), and the retardation parameter $(\beta)$. One of the aims of this work is to analyse the distribution of axial velocity and maximum axial velocity across and along the channel as a function of $L^{2}$ and $\beta$, under the presence of strong inertial and elastic forces. To accomplish this, we have done numerical simulations at the same three Reynolds numbers $(R e=486,532$ and 583), for Weissenberg numbers ranging from 0.1 to 1.0 , for different $L^{2}(50,100$ and 200$)$ and $\beta$ ranging from 0.9 to 0.1 . To analyse the effect of extensibility, $\beta$ was fixed at 0.5 , whereas to analyse the effect of the retardation parameter, the extensibility was set to $L^{2}=100$. The results are presented for different positions along the curve and across the duct square cross-section, through transverse profiles and contour plots of the streamwise velocity $(U)$, and vector plots of the secondary flow, as well as through the variation of maximum axial velocity $\left(U_{\max }\right)$ with the different controlling parameters. The velocity profiles are extracted at specific values of the $Z$ coordinate of the cross-section, most at the mid-plane $Z=0.5$ (cf. Fig. 4a), and the velocity contours and vector plots of the secondary flow are extracted at different angular positions along the curve, as illustrated in Fig. 4b.

\subsection{Viscoelastic simulations}

As mentioned previously, at the inlet of the domain ( $x=0$, a distance of $20 a$ upstream of the curve entrance) a fully developed flow was imposed based on an analytical solution valid for both the 

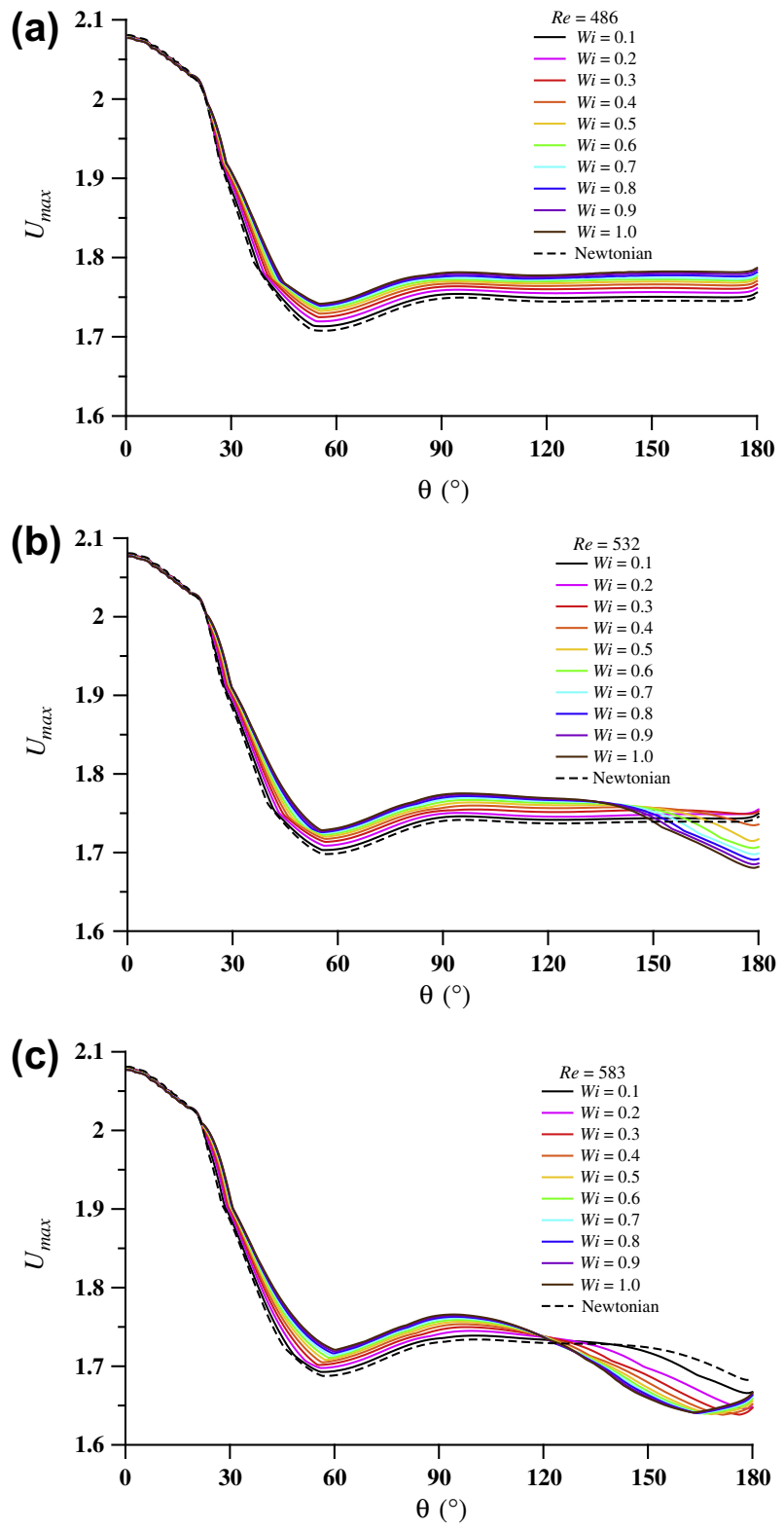

Fig. 8. Variation of maximum axial velocity $U_{\max }$ with $W i$ along the curve for (a) $R e=486$, (b) $R e=532$ and (c) $R e=583$, with $L^{2}=100$ and $\beta=0.5$.

Newtonian and the FENE-CR fluids. Fig. 5 shows the profiles of streamwise velocity across the section at the entrance $\left(\theta=0^{\circ}\right)$ to the curve (Fig. 5a) and the corresponding contour plot (Fig. 5b). The profiles are parabolic and perfectly symmetric about the middle $Z=0.5$ plane (Fig. $5 a$ ), and the contour plot (Fig. 5b) shows the maximum velocity right in the middle of the cross-section and that the velocity profiles are symmetrically distributed across the section. The case presented is for $R e=486, W i=0.1, L^{2}=100$ and $\beta=0.5$, but the same pattern was verified for all cases simulated.

Fig. 6 shows the variation of the axial velocity $(U)$ along the curve, with the curvature coordinate given by the angle $\theta$, at the middle plane $Z=0.5$ (cf. Fig. 4), as a function of $W i$ for $R e=532$, $L^{2}=100$ and $\beta=0.5$. It is noted that the present predictions for the development of the axial velocity along the channel are rather similar to those for the Newtonian fluid measured by Bara et al. [6]. In the initial part of the curve and up to $\theta=90^{\circ}$ (Fig. 6a-c) the profiles are identical and independent of $W i$. The flow runs against the outer wall of the curve, exhibiting a sharp peak near this wall because of inertia. As a consequence of mass conservation the axial
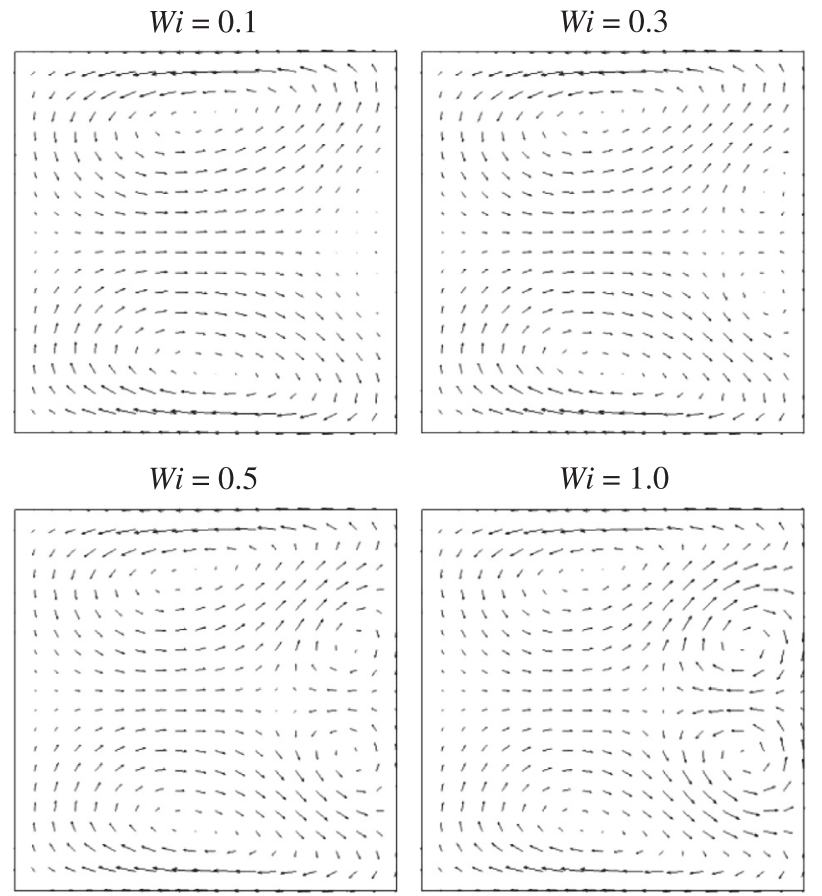

Fig. 9. Variation of secondary flow pattern with $W i$, for $R e=532, \beta=0.5$ and $L^{2}=100$, at position $\theta=150^{\circ}$.

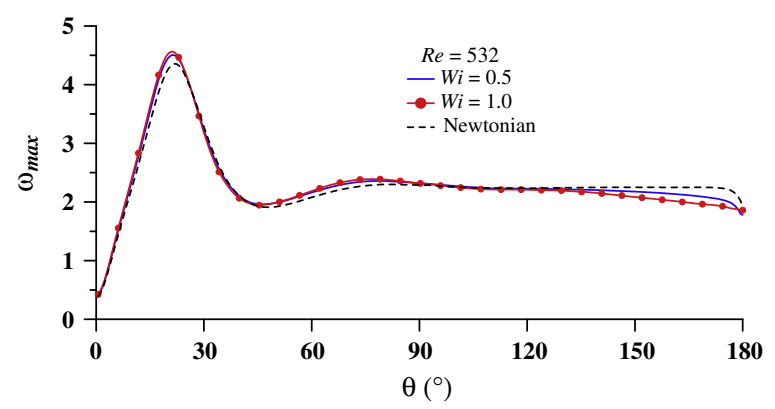

Fig. 10. Variation of maximum vorticity $\left(\omega_{\max }\right)$ intensity along the curve with $W i=0.5$ and 1.0 for $R e=532$, with $L^{2}=100$ and $\beta=0.5$, and variation of its position in the cross-section for the case $W i=0.5$.

velocity near the inner wall decreases and a secondary flow sets in at the cross section of the duct, characterised by a pair of symmetrical counter rotating vortices, as shown in the vector plots of Fig. 7. On moving downstream along the curve (Fig. 6a and b), the peak velocity smoothes out and slightly moves toward the channel centre and, as a consequence, the axial velocity near the inner wall increases. This redistribution of velocity is a result of the secondary flow transporting high momentum fluid from the outer wall region to the inner wall region (Fig. 7). In addition, Fig. 6 shows that for this level of inertia $(R e=532)$ elasticity only affects the velocity distribution in the second half of the curve $\left(\theta>90^{\circ}\right.$, Fig. $6 \mathrm{~d}$ and $\left.\mathrm{f}\right)$, and that momentum transfer of axial velocity towards the centre of the channel increases with $W i$.

The variation of maximum axial velocity $\left(U_{\max }\right)$, which is a consequence of the formation and development of the secondary flow, is depicted in Fig. 8, with variation of Wi and considering different $R e$. For all cases simulated the variation of maximum velocity follows the same trend in the first half of the curve (up to $\theta=90^{\circ}$ ): the maximum axial velocity has its highest value at the entrance of the channel $\left(\theta=0^{\circ}\right)$, decreasing around $20 \%$ along the curve up to $\theta=60^{\circ}$ as the secondary flow sets in and starts to distribute 

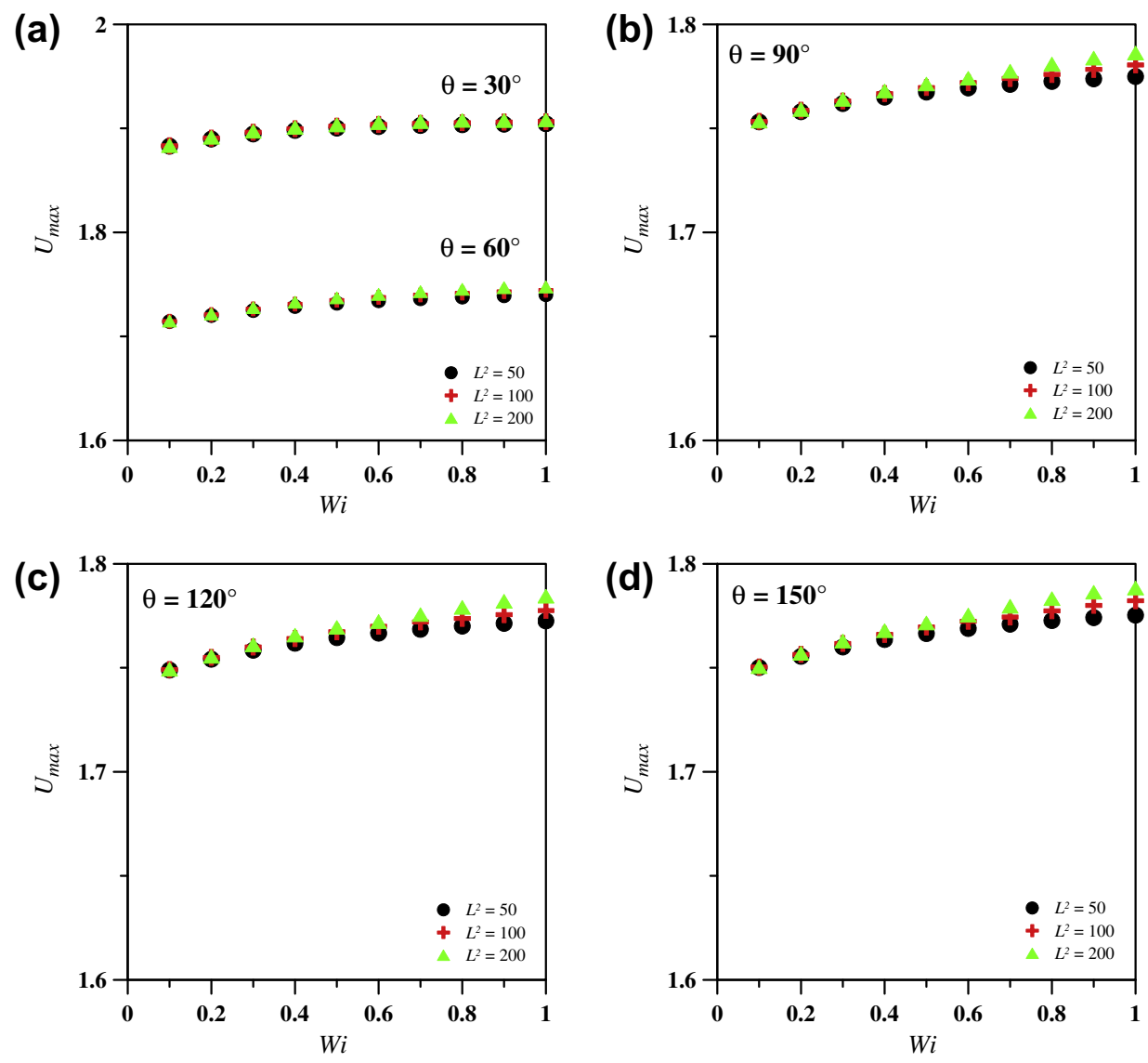

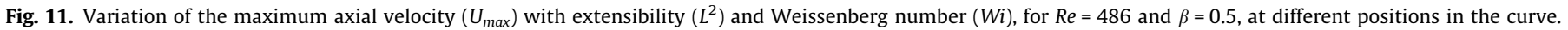
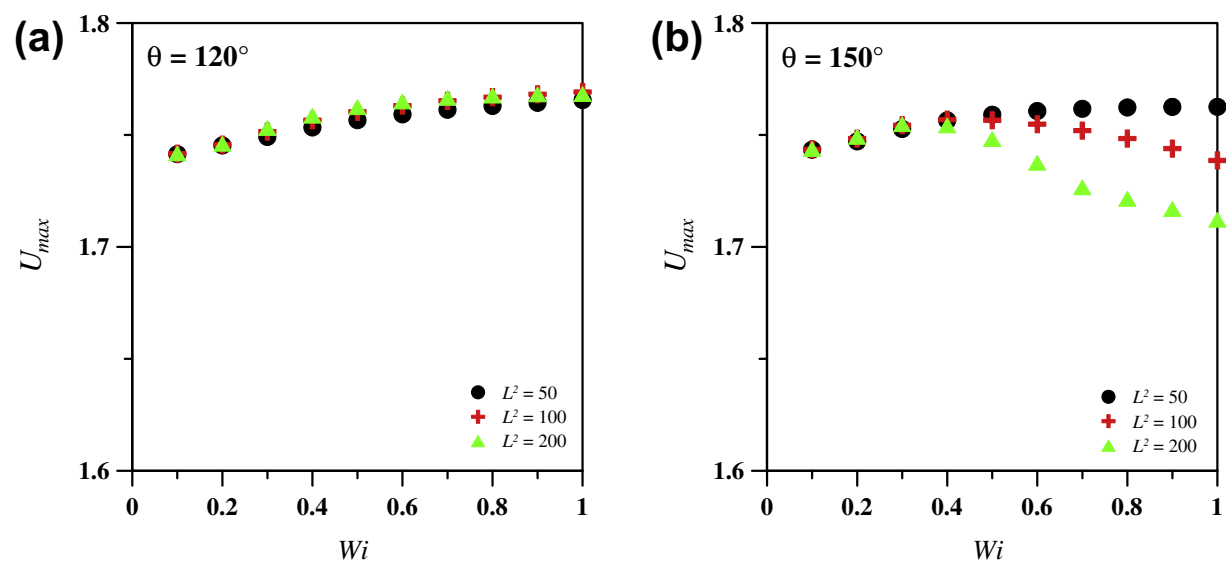

Fig. 12. Variation of maximum axial velocity $\left(U_{\max }\right)$ with extensibility $\left(L^{2}\right)$ and Weissenberg number $(W i)$, for $R e=532$ and $\beta=0.5$, at positions $($ a $) 120^{\circ}$ and $(\mathrm{b}) 150^{\circ}$.

momentum to the whole cross-section. Downstream of this position, the velocity peak increases until the middle of the curve $\left(\theta=90^{\circ}\right)$, but the variations are now much smaller than at the beginning. This pattern is observed for the various sets, all pertaining to conditions of strong inertia (Reynolds numbers of 486, 532 and 583), but nevertheless the effects of elasticity are observed from, approximately, $\theta=30^{\circ}$, where the reduction of velocity is higher for smaller values of Wi. Such effects may be understood by considering that elasticity tends to oppose inertial effects and keep, for a longer distance on entering the curve, the initial velocity conditions.

In the second half of the curved channel elastic effects accentuate secondary flow formation, get stronger and become distinct for each Re case considered. Downstream of $\theta=90^{\circ}$, at the lowest Reynolds number of 486 (Fig. 8a), the maximum velocity tends to a constant value for each $W i$, so the effect of elasticity is minimum, but as the Reynolds number is progressively increased (Fig. 8b and c) we observe a second drop in the peak velocity at the end of the curve, which is intensified by flow elasticity. This reduction of $U_{\max }$ in the second portion of the curve is related to the onset and growth of an additional pair of counter rotating vortices. The vector plot of Fig. 9 depicts the appearance of this second pair of vortices near the outer wall with increasing $W i$ at position $\theta=150^{\circ}$ for $R e=532$, whereas the plot of the streamwise velocity in Fig. 6e, taken at the mid-plane of the channel, shows the increasing momentum transfer from the outer wall towards the centre of 


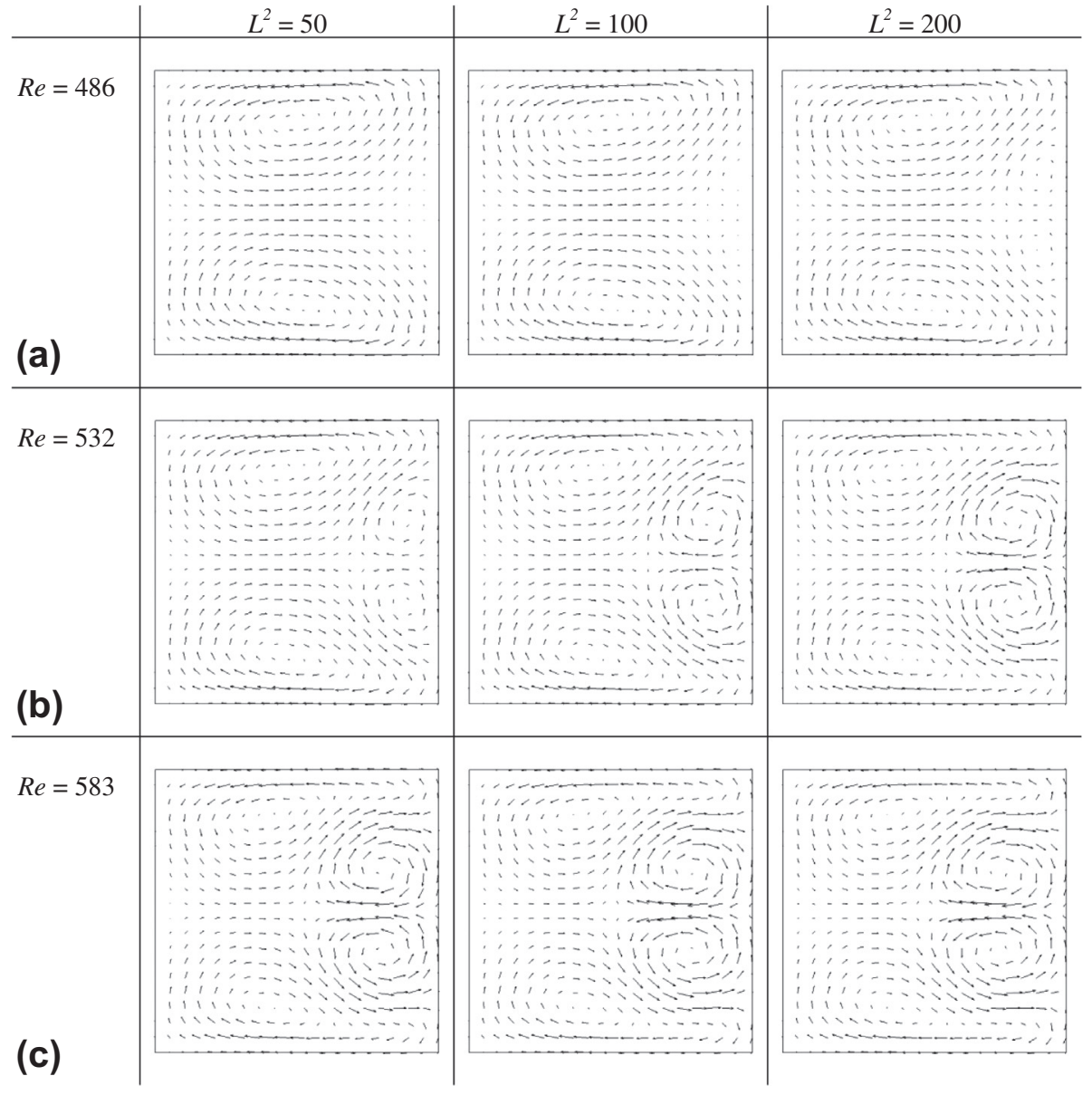

Fig. 13. Variation of secondary flow pattern with $L^{2}$, for (a) $R e=486$, (b) $\operatorname{Re}=532$ and (c) $\operatorname{Re}=583$. All with $\beta=0.5$ and $W i=1.0$ at position $\theta=150^{\circ}$.
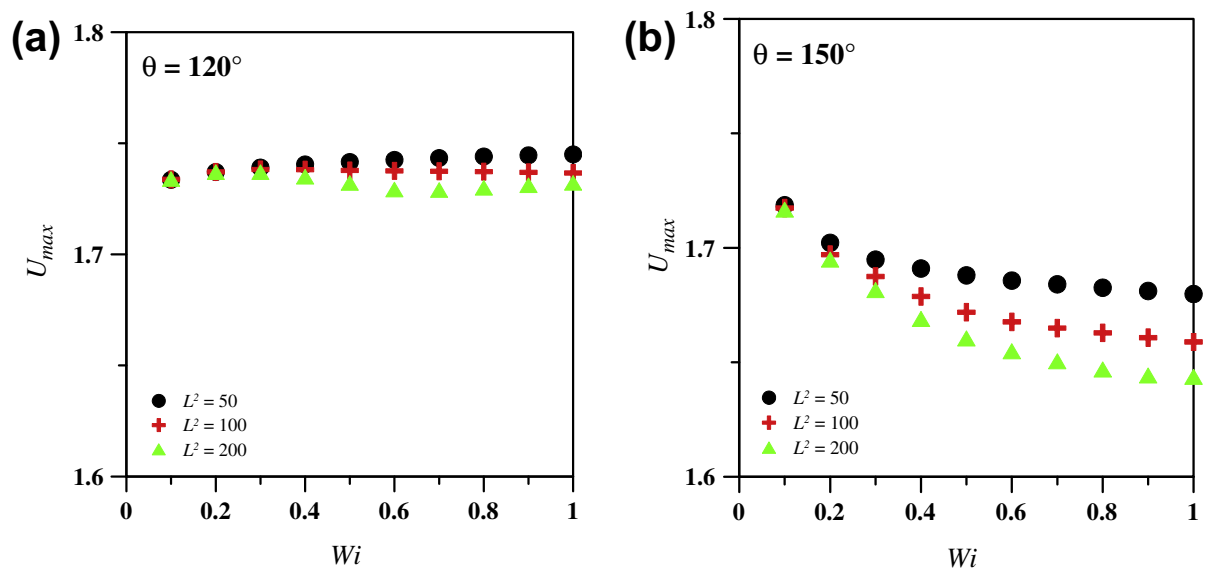

Fig. 14. Variation of the maximum axial velocity $\left(U_{\max }\right)$ with extensibility $\left(L^{2}\right)$ and Weissenberg number $(W i)$ for $R e=583, \beta=0.5$, at positions $($ a $) 120^{\circ}$ and $(\mathrm{b}) 150^{\circ}$.

the channel with increasing $W i$. For $W i=0.1$ the additional pair of vortices is not formed, but at $W i=0.3$ it already appears (turning point where $U_{\max }$ starts to decrease in the second part of the curve in Fig. 8b, and the momentum transfer to the centre of the channel is significant, Fig. 6e), although these vortices are still small in size. Further increasing $W i$ the additional pair of vortices increases in size (Fig. 9) leading to a higher decrease in $U_{\max }$ (Fig. 8b) and to a higher transverse transfer of streamwise momentum towards the centre of the curved channel (Fig. 6e).

A way of visualising the intensity of the centrifugally-generated secondary flow is provided by the maximum value of the stream- wise component of the vorticity, which is shown along the curve in Fig. 10 for the Newtonian and two viscoelastic cases (with $W i=0.5$ and 1.0 , for $\beta=0.5$ and $L^{2}=100$ ). We note that for the present three-dimensional developing flow it is not possible to define a streamfunction in the cross-sectional plane that would indicate more directly the amount of secondary flow.

There are two locations in the cross-section having $\omega_{X}= \pm \omega_{\max }$, which reside near the eye of each counter-rotating vorticity cell formed symmetrically to the mid-plane $Z=0.5$ as a consequence of the spiraling motion induced by the curve. Elasticity is seen to promote faster vorticity intensification initially (up to $\theta=20^{\circ}$, an 

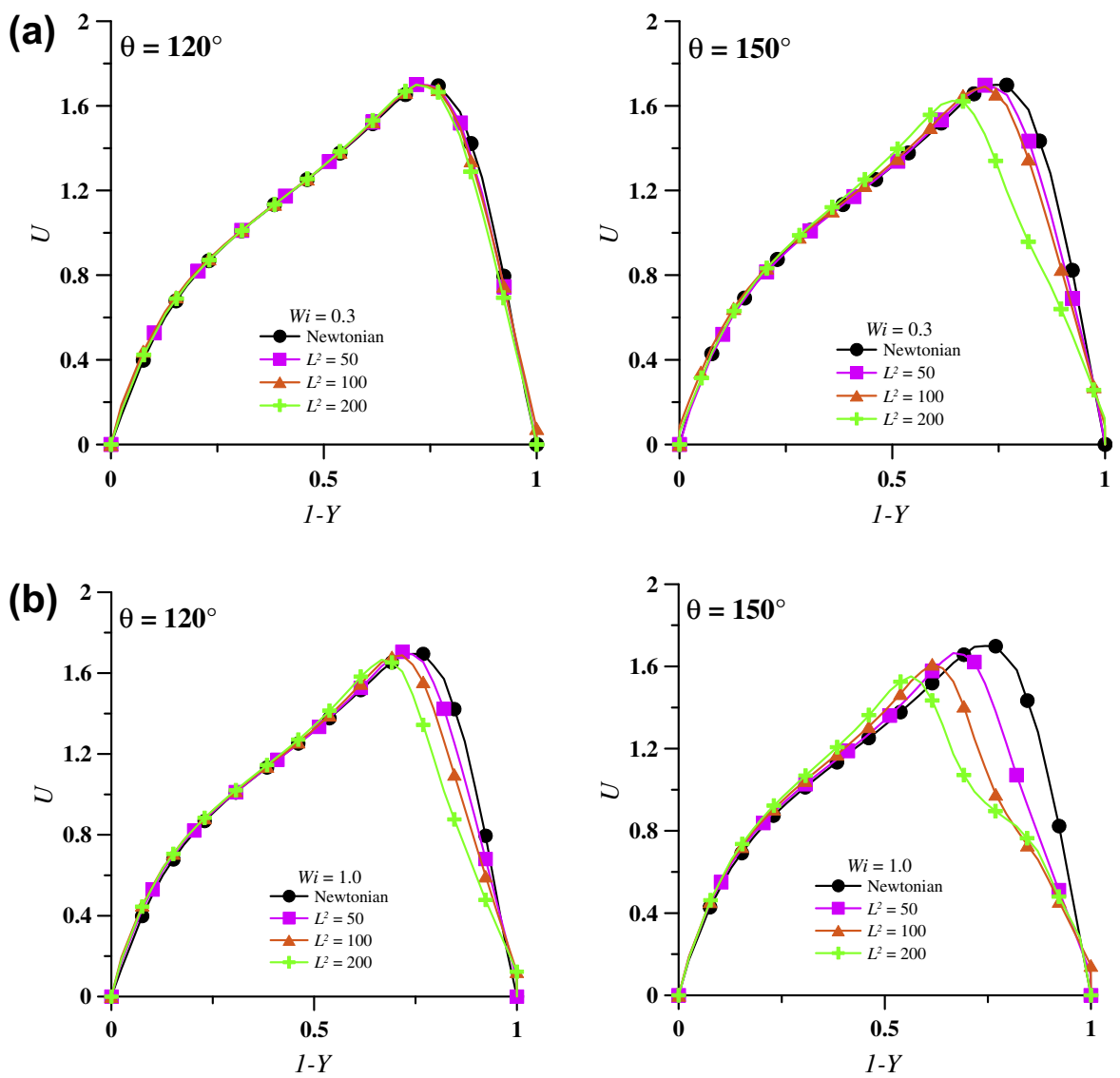

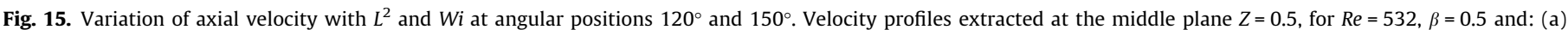
$W i=0.3$; (b) $W i=1.0$.
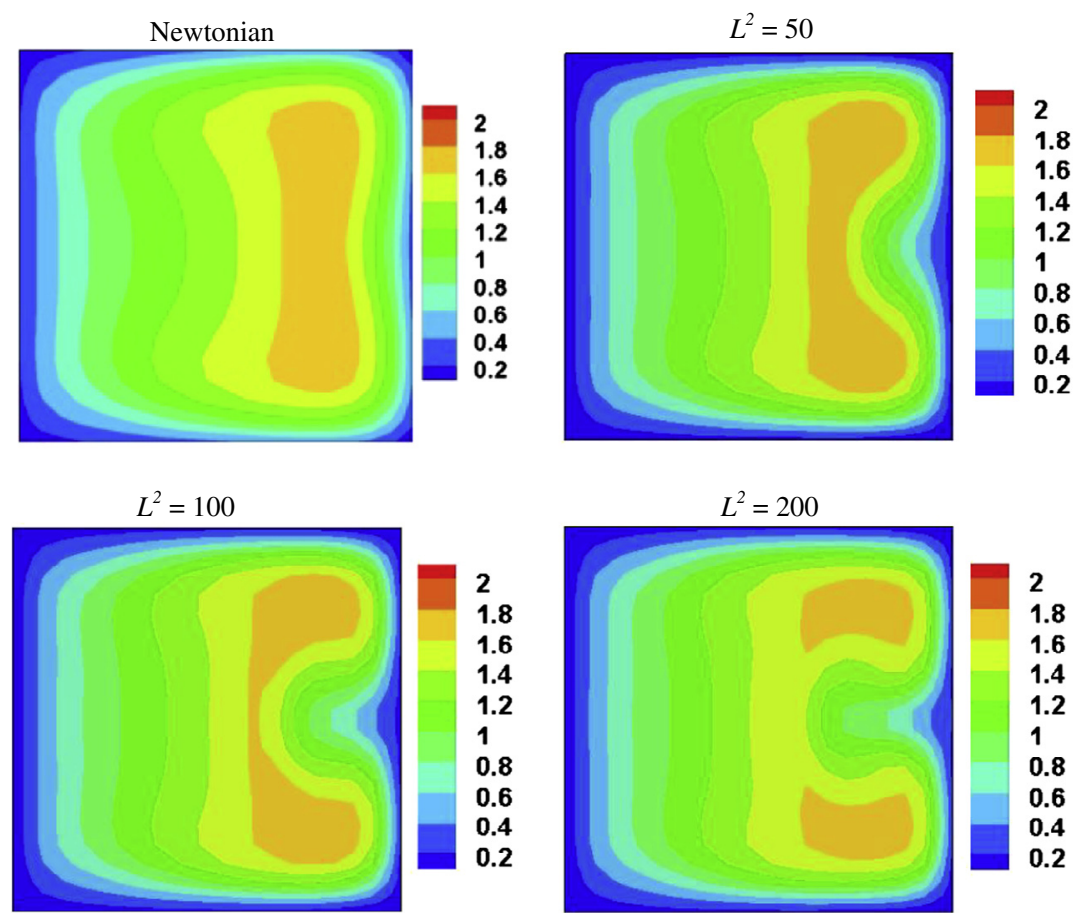

Fig. 16. Variation of the axial velocity distribution $(U)$ with $L^{2}$, for $R e=532, W i=1.0$ and $\beta=0.5$, at location $\theta=150^{\circ}$.

history-like effect) and a faster (elastic) recovery up to the end of the first part of the curve $\left(40^{\circ} \leqslant \theta \leqslant 90^{\circ}\right)$, although the differences compared to the Newtonian evolution are not very significant. Then, in the second part of the curve $\left(90^{\circ} \leqslant \theta \leqslant 180^{\circ}\right)$, the Newto- 

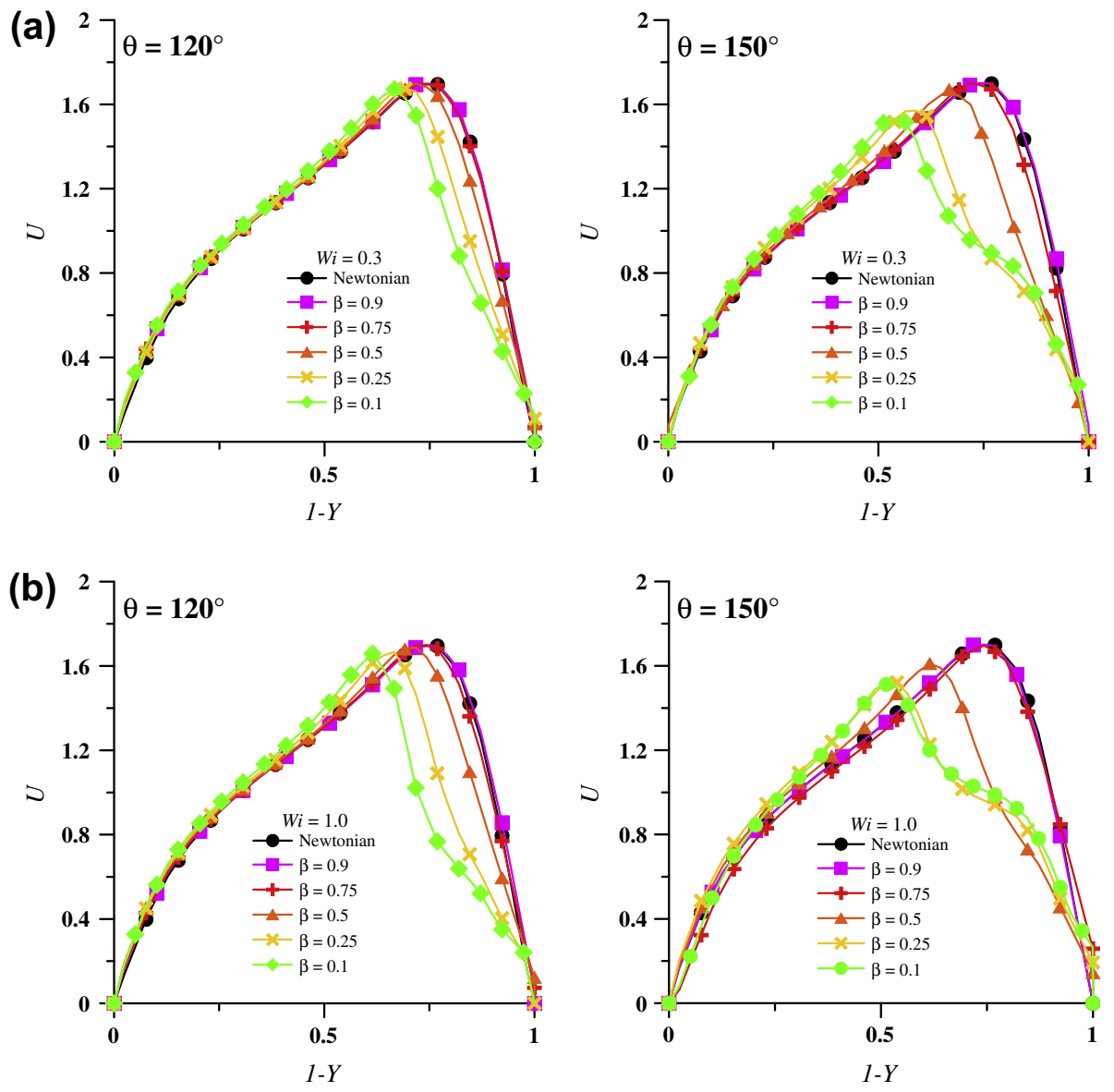

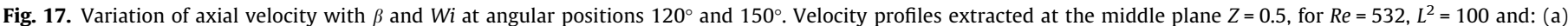
$W i=0.3$; (b) $W i=1.0$.

nian solution attains a fully-developed situation $\left(\omega_{\max } \cong C t e=2.3\right)$ while the viscoelastic starts decreasing after $\theta \approx 120^{\circ}$, due to the formation and intensification of the second pair of vortices, as discussed before (Fig. 9).

\subsection{Effect of polymer extensibility}

Parameter $L^{2}$ quantifies the stretch of the polymer molecules (via their simplified representation, the dumbbells) within the fluid. As extensibility increases the polymer molecules tend to reach a fully stretched state and in extreme cases, such as UCM and Oldroyd-B fluid models, extensibility is $L^{2}=\infty$. Fig. 11 presents the variation of maximum axial velocity $\left(U_{\max }\right)$ with Weissenberg number and extensibility, for $R e=486$ and at different angular positions along the curve $\left(\theta=30^{\circ}, 60^{\circ}, 90^{\circ}, 120^{\circ}\right.$ and $\left.150^{\circ}\right)$. It is seen that the maximum axial velocity $\left(U_{\max }\right)$ increases with both $W i$ and $L^{2}$ as $\theta$ grows and, after a reduction in the level of $U_{\max }$ between $30^{\circ}$ and $60^{\circ}, U_{\max }$ still grows slightly with elasticity as one progresses along the curved duct. Nevertheless, the effect of extensibility is only evident for $W i>0.5$ and $\theta>60^{\circ}$. By increasing inertia from $R e=486$ to 532 the same behaviour is observed initially, but then (Fig. 12), at position $120^{\circ}, U_{\max }$ slightly decreases for $L^{2}=200$ and $W i>0.6$ (comparing with Fig. $11 \mathrm{c}$ for $R e=486$ at the same position). This behaviour is prominent at the end of the curve $\left(\theta=150^{\circ}\right.$, Fig. 12b), where $U_{\max }$ decreases with extensibility, but for $W i>0.3$. This is actually a consequence of the appearance and growth of the second pair of counter rotating vortices near the outer wall, as is well illustrated in Fig. 13b. Here one must compare these vector plots with those pertaining to the lower Re flows of
Fig. 13a, for which the peak velocity does not decrease (Fig. 11d) and the second pair of counter rotating vortices does not exist. Actually, looking closer to Fig. 13a, the additional pair of counter rotating vortices is only incipient for the highest extensibility case $\left(L^{2}=200\right)$, but it is not strong enough to reverse the variation of $U_{\max }$ (Fig. 11d), as occurs at higher inertia in Fig. 12b. As inertia increases the effect of $L^{2}$ becomes more pronounced as seen in Fig. 14 for $R e=583$. Here the variation of the peak velocity with $L^{2}$ is larger than at lower $R e$ and, for $\theta>90^{\circ}, U_{\max }$ decreases more intensely with $W i$ than at lower Re. As explained above these variations are concomitant with the growth of the additional second pair of vortices near the outer wall (Fig. 13c), promoted by extensibility.

The velocity distribution in the cross-section proved to be always top-bottom symmetric ( $z$ direction) and the velocity distribution and its development is like the one described and illustrated in Fig. 6. In agreement with what was observed regarding the variation of the velocity field with $W i$, in the first half of the curve the flow is not affected by the polymer extensibility, but further downstream the transport of maximum momentum seen to occur in the middle plane from the outer wall region towards the centre of the channel increases with $L^{2}$ (Fig. 15a, with $W i=0.3$ ). The same features take place with increasing intensity for higher elasticity (Fig. 15b, with $W i=1.0$ ). For $W i=1.0$ (Fig. 15b), the increase in momentum transfer starts earlier in the curve (differences are clear from $120^{\circ}$ onwards) as $L^{2}$ is increased. The momentum transfer in the middle plane is another consequence of the changes in the secondary flow due to molecular extensibility variations. The contours of axial velocity (Fig. 16) and the vector 


$$
\theta=120^{\circ}
$$
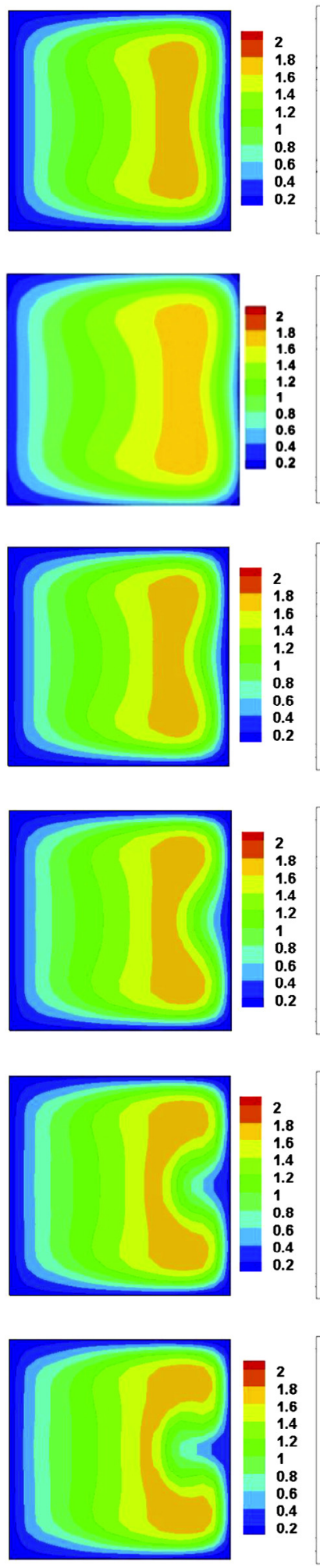
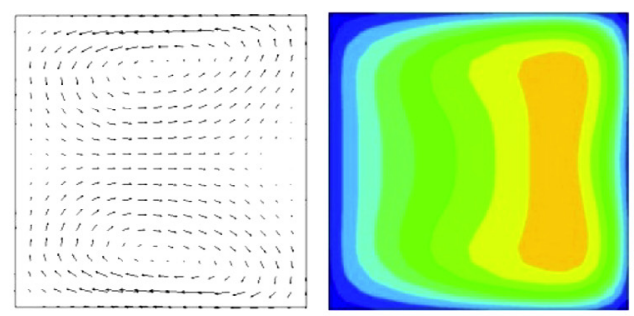

$\theta=150^{\circ}$

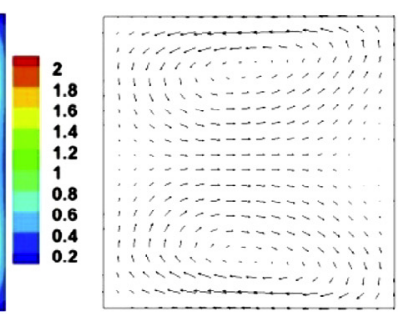

Newtonian
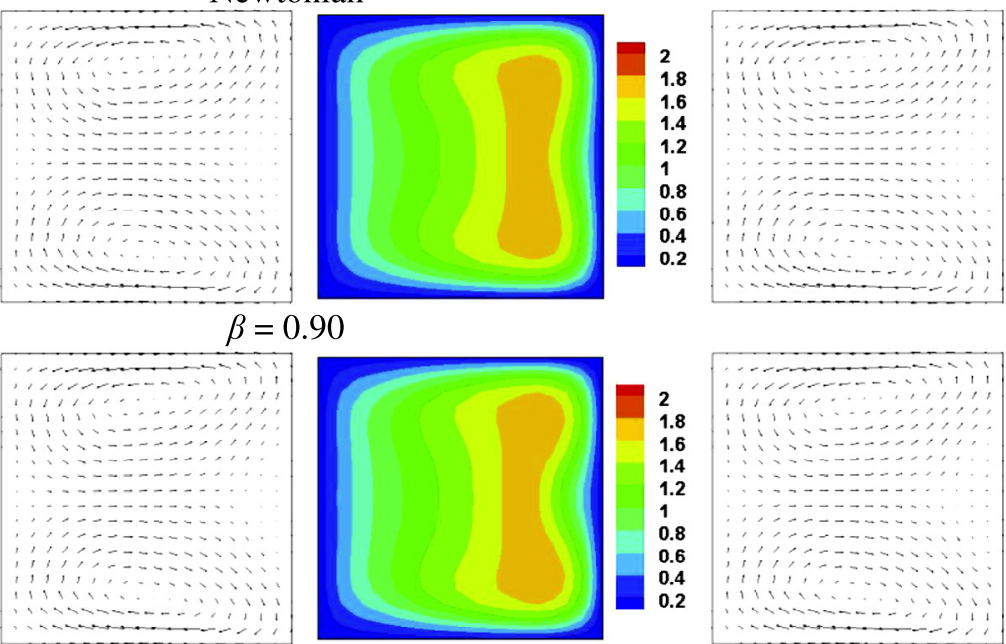

$\beta=0.75$
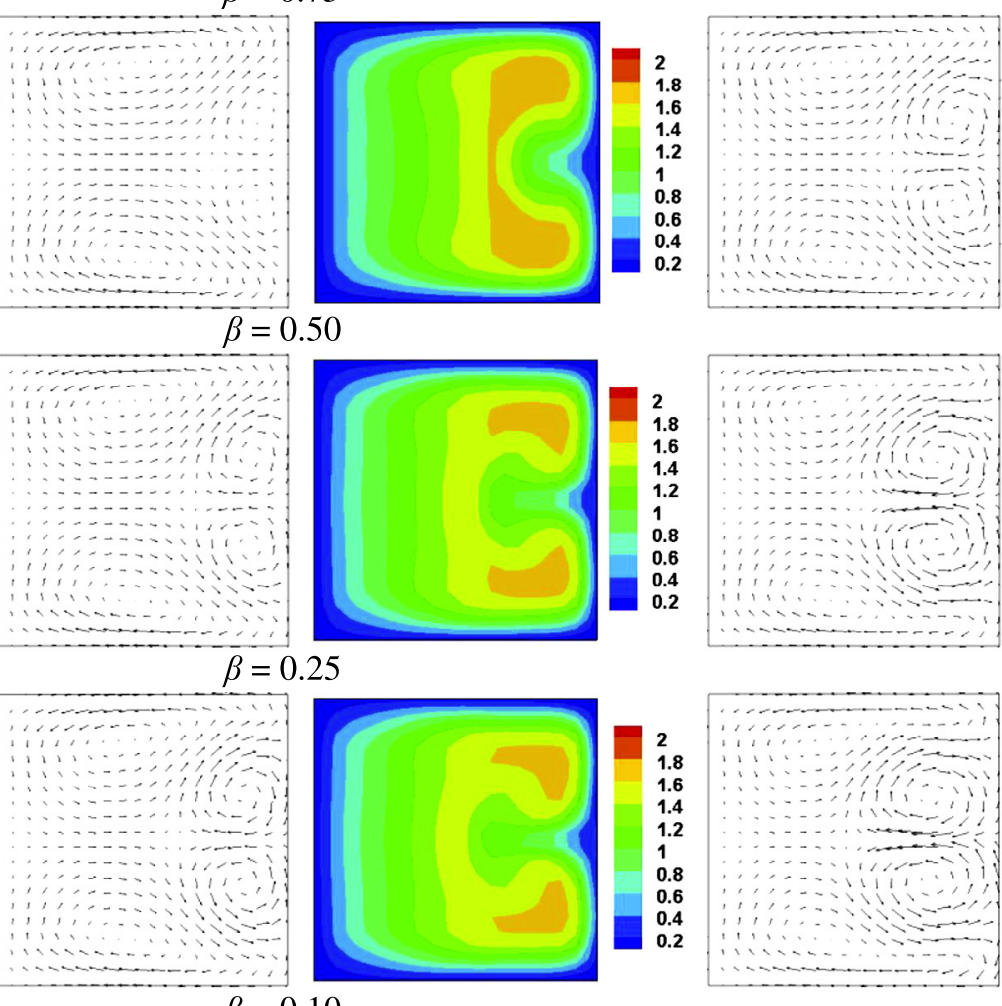

$\beta=0.10$

Fig. 18. Axial velocity contours and secondary flow patterns as a function of $\beta$, for $R e=532, W i=1.0$ and $L^{2}=100$, at positions $120^{\circ}$ and $150^{\circ}$.

plots of the secondary flow in Fig. 13b show these changes in the patterns for the cases in Fig. 15b, at position $150^{\circ}$. For Newtonian flow there is a region of maximum velocity near the outer wall
(Fig. 16 left) and the secondary flow is characterised by a single pair of counter rotating vortices (e.g. Fig. 9 at $W i=0.1$ ). On the other hand, the flow of viscoelastic fluids, which have been shown 
(a)

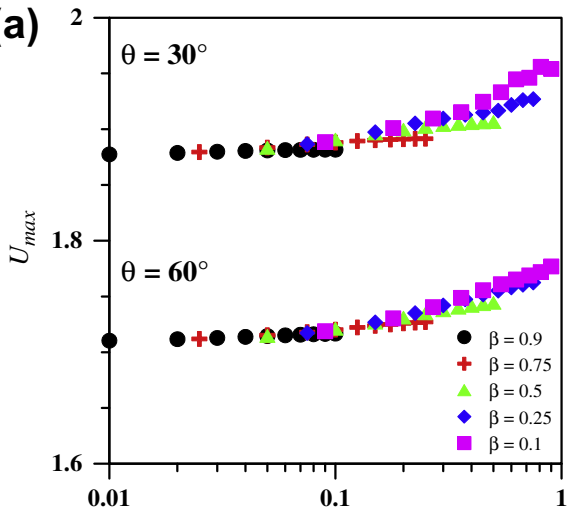

(1- $\beta) W i$



$(1-\beta) W i$

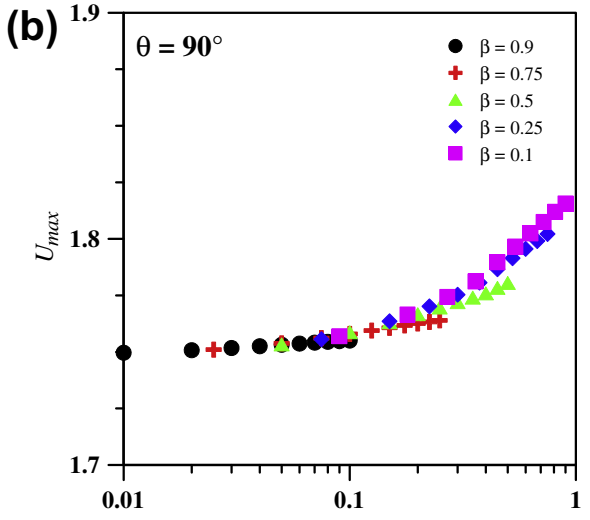

(1- $\beta) W i$

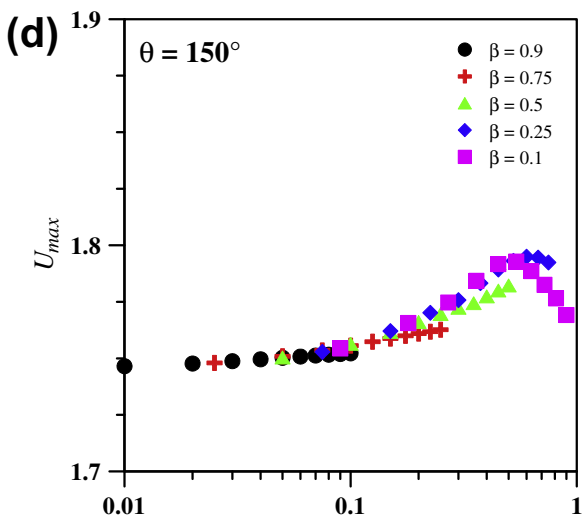

(1- $\beta) W i$

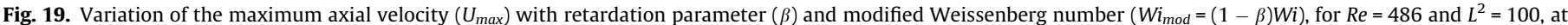
different positions in the curve.

(a)

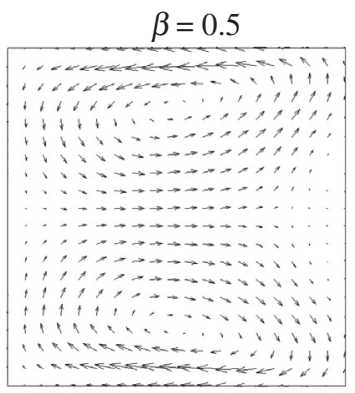

(b)

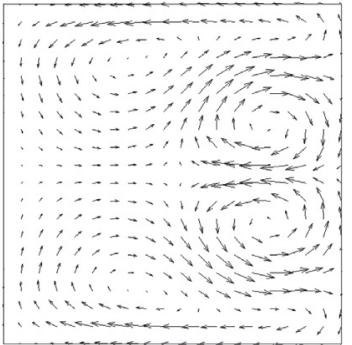

$\beta=0.25$
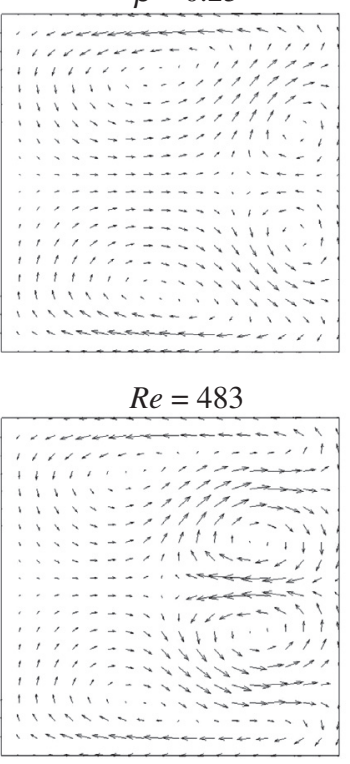

$R e=583$
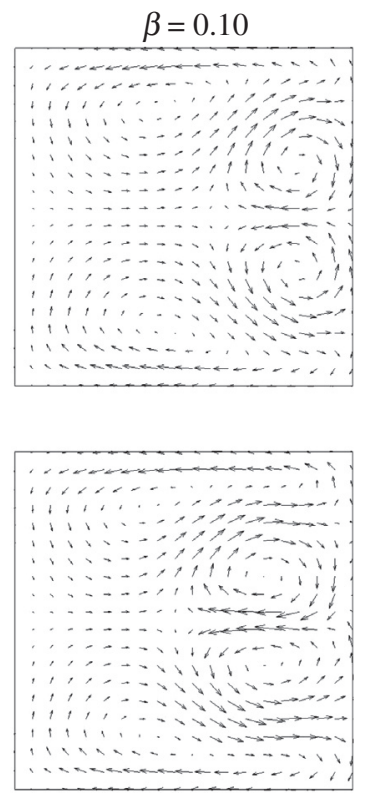




$(1-\beta) W i$

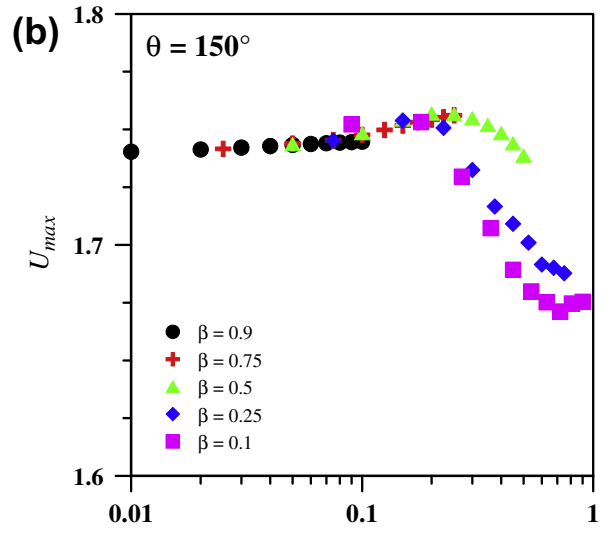

$(1-\beta) W i$

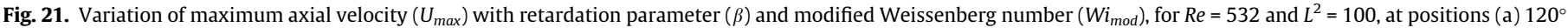
and (b) $150^{\circ}$.
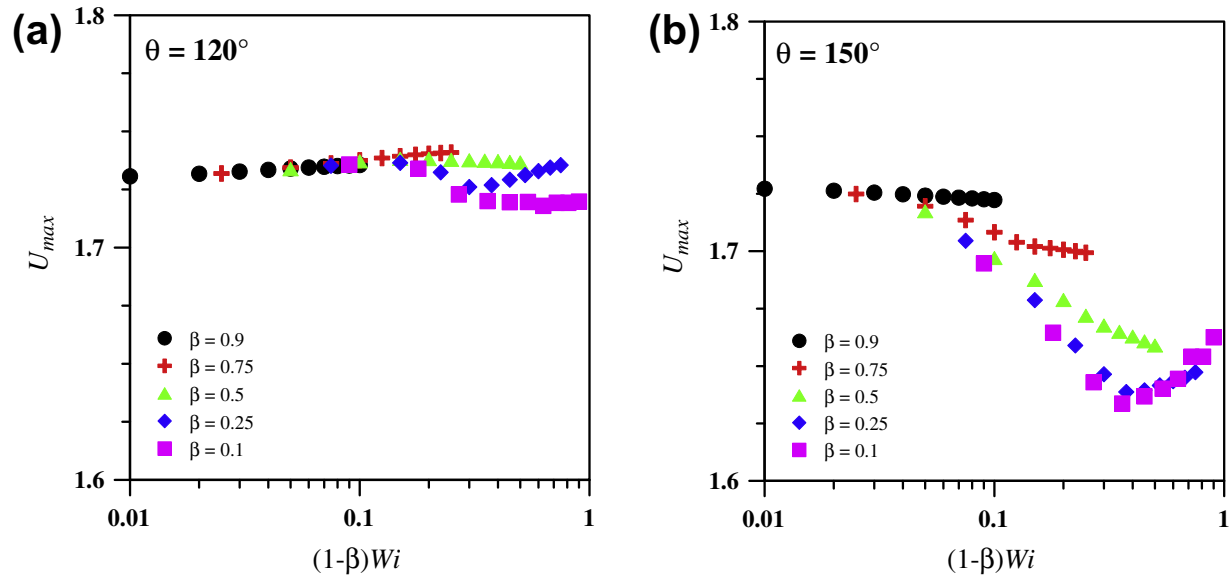

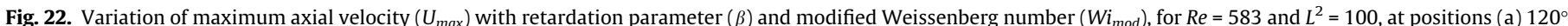
and (b) $150^{\circ}$

\subsection{Effect of retardation parameter}

As defined previously, the retardation parameter is the relation of two different times, the relaxation time (which is the time needed by the polymer chains to relax to their equilibrium state after the application of a stress has ceased) and the retardation time (which is a fraction of the relaxation time dependent on solvent viscosity ratio) [24]. Alternatively, the retardation parameter can also be defined as a relation of viscosities, the ratio of the solvent viscosity to the total viscosity (sum of solvent and polymer viscosities), i.e., $\beta$ is expressed as $\beta=\eta_{s} / \eta=\lambda_{r} / \lambda$. When $\beta$ is 1 we have the Newtonian case, but as $\beta$ decreases elasticity effects become stronger and flow development is expected to become similar to that observed when varying $W i$ and $L^{2}$.

The distribution and development of the axial velocity along the middle plane of the cross-section followed a similar general behaviour as that reported when describing the variation with $W i$ and $L^{2}$, in Figs. 6 and 15, respectively. In this case the momentum transfer besides increasing with $R e$ and $W i$ also increases with $(1-\beta)$, as depicted in Fig. 17. Comparing with the cases of Fig. 15, which dealt with the influence of $L^{2}$, the effects associated with elasticity start earlier along the curve and exhibit higher intensity, especially at low $\beta$, than shown before while analysing the combined effect of extensibility and Wi. Again and as previously discussed, this momentum transfer is directly related to the formation of the additional pair of counter rotating vortices, which redistribute the axial velocity across the section (Fig. 18). Fig. 18 illustrates the effect of the retardation parameter on the distribution of axial velocity in the cross section and also on the secondary flow pattern, at locations $120^{\circ}$ and $150^{\circ}$. It shows that the maximum streamwise velocity is maintained near the outer wall of the curve (as in every case simulated). Also, the momentum transfer, which occurs in the middle region of the cross-section and is higher with decreasing $\beta$ (Fig. 17), is the result of the development of the second pair of counter rotating vortices near the outer wall, leading to the separation of the region of maximum velocity near the outer wall (Fig. 18). The second pair of vortices is visible for $\beta<0.75$ and its size increases downstream along the curve and also as the parameter $\beta$ is reduced. It is remarked that the flow is always symmetric with respect to the plane $Z=0.5$.

Finally, the variation of the maximum axial velocity $\left(U_{\max }\right)$ with $\beta$ and with the modified Weissenberg number $\left(W i_{m o d}\right)$ for various $R e$ is shown in Figs. 19-22. Similarly to what was seen in Fig. 11 while studying the influence of $W i$ and $L^{2}$, here, when elasticity is increased by decreasing the retardation parameter there is an increase in the maximum velocity over the whole length of the channel (Fig. 19), except at $150^{\circ}$, where the maximum axial velocity for $\beta=0.25$ and 0.1 starts to decrease for $W i_{\text {mod }}>0.6$ (Fig. 19d). Previously, the same behaviour was observed in flows with higher inertia $(R e=532$ in Fig. 12), when the variation of extensibility was investigated. Fig. 20 shows that for lower $\beta(0.25$ and 0.1$)$, the additional pair of vortices appears at $R e=486$, which corresponds to 
the decrease in $U_{\max }$ seen in Fig. 19d. At higher values of Re (Fig. 21, with $R e=532$ ), the decrease in $U_{\max }$ at position $150^{\circ}$ takes place at lower elasticity $\left(W i_{\text {mod }}=0.2\right)$ compared to the $R e=486$ case of Fig. 19; in addition, for $R e=532$ this reduction of $U_{\max }$ starts occurring earlier in the curve $\left(120^{\circ}\right)$ for lower $\beta$. However, for $R e=583$ (Fig. 22), after decreasing, the variation of maximum axial velocity reverses and starts to increase for $\beta<0.5$ at the end of the curve.

\section{Conclusions}

The FENE-CR allows the investigation of elastic effects in the absence of viscosity shear-thinning effects that often exist in real fluids and in various other constitutive equations. Using this model, this work investigates the effects of elasticity upon the flow in a curved channel with a square cross-section under conditions of non-negligible inertia. Specifically, the influence of Reynolds number in combination with polymer extensibility $\left(L^{2}\right)$, retardation parameter $(\beta)$ and flow elasticity $(W i)$ are analysed in detail for Reynolds numbers on the range 486-583.

In the first half of the curve (up to $90^{\circ}$ ) the flow is inertia dependent, and none of the parameters quantifying elasticity affected flow development in the ranges investigated. Here, inertia together with centrifugal forces are responsible for pushing and maintaining maximum velocities near the outer wall generating an uneven velocity distribution, which is tightly connected to the development of the secondary flow in the cross section, characterised by a pair of counter rotating vortices spanning the whole width of the channel. In the second half of the curve, flow development is affected by fluid elasticity and each relevant dimensionless number quantifying fluid elasticity has the same qualitative effect. Increasing $L^{2}$, increasing $W i$ or decreasing $\beta$ all lead to the appearance of an additional pair of counter rotating vortices near the outer wall at lower $R e$ than in the absence of elasticity. This phenomenon is responsible for the transfer of momentum from the region of maximum momentum near the outer wall to the centre of the channel where momentum is lower, consequently reducing the magnitude of the maximum axial velocity magnitude.

The flow in curved channels is highly dependent on geometry, and caution is necessary when comparisons are made between results. For the same geometry, the onset of the additional pair of vortices was also verified by Helin et al. [19] to be dependent on inertia and elasticity for a modified PTT model and an Oldroyd-B fluid model with $\beta=1 / 9$. The development of the additional pair of vortices was observed for the modified PTT fluid at a Deborah number of 0.4 for $R e=486$, but for an Oldroyd-B fluid was only reported to take place at $R e=583$. Our results showed that for $R e=486$, the additional pair of vortices is observed for lower $\beta$ and $W i>0.7$. These results confirm findings by Helin et al. [19] in allowing us to say that the presence of shear-thinning acts in the same direction as fluid elasticity in that it also favours the transition from a secondary flow with one to two pairs of vortices, at lower inertia.

\section{Acknowledgements}

Funding by Fundação para a Ciência e Tecnologia (Portugal) under Grant SFRH/BD/68357/2010 and Project PTDC/EME-MFE/ $114322 / 2009$ of COMPETE Program are gratefully acknowledge.

\section{References}

[1] S.A. Berger, L. Talbot, L.-S. Yao, Flow in curved pipes, Annu. Rev. Fluid Mech. 15 (1983) 461-512.

[2] S. Vashisth, V. Kumar, K.D.P. Nigam, A Review on the potential applications of curved geometries in process industry, Ind. Eng. Chem. Res. 47 (2008) 32913337.

[3] J. Eustice, Experiments on stream-line motion in curved pipes, Proc. Royal Soc. A: Math. Phys. Eng. Sci. 85 (1911) 131-199.

[4] W.R. Dean, Note on the motion of fluid in a curved pipe, Philos. Mag. 4 (20) (1927) 208-223

[5] H.G. Cuming, The secondary flow in curved pipes, Aeronaut. Res. Counc. Rep. Memo. 2880 (1952).

[6] B. Bara, K. Nandakumar, J.H. Masliyah, An experimental and numerical study of the Dean problem: flow development towards two-dimensional multiple solutions, J. Fluid Mech. 244 (1992) 339-376.

[7] H. Fellouah, C. Castelain, A. Ould-El-Moctar, H. Peerhossaini, The Dean instability in power-law and Bingham fluids in a curved rectangular duct, J. Non-Newton. Fluid Mech. 165 (2010) 163-173.

[8] Y. Iemoto, M. Nagata, F. Yamamoto, Steady laminar flow of viscoelastic fluid in a curved pipe of circular cross-section with varying curvature, J. Non-Newton. Fluid Mech. 22 (1) (1986) 101-114.

[9] B. Das, Flow of a Bingham fluid in a slightly curved tube, Int. J. Eng. Sci. 30 (1992) 1193-1207.

[10] N. Phan-Thien, R. Zheng, Viscoelastic flow in a curved channel: a similarity solution for the Oldroyd-B fluid, J. Appl. Math. Phys. 41 (1990) 766-781.

[11] Y.L. Joo, S.G. Shaqfeh, Viscoelastic Poiseuille flow through a curved channel: a new elastic instability, Phys. Fluids A 3 (9) (1991) 2043-2046.

[12] Y.L. Joo, S.G. Shaqfeh, A purely elastic instability in Dean an Taylor-Dean flow Phys. Fluids A 4 (3) (1992) 524-543.

[13] Y.L. Joo, S.G. Shaqfeh, The effects of inertial on the viscoelastic Dean an TaylorCouette flow instabilities with application to coating flows, Phys. Fluids A 4 (11) (1992) 2415-2431

[14] V.B. Sarin, The steady laminar flow of an elastic-viscous liquid in a curved pipe of varying elliptic cross-section, Math. Comput. Model. 26 (1997) 109-121.

[15] M.-K. Zahng, X. -R Shen, J.-F. Ma, B.-Z. Zhang, Flow of Oldroyd-B in rotating curved square ducts, J. Hydrodyn. 19 (1) (2007) 36-41.

[16] M. Norouzi, M.H. Kayhani, C. Shu, M.R.H. Nobari, Fow of second-order fluid in a curved duct with square cross-section, J. Non-Newton. Fluid Mech. 165 (2010) 323-339.

[17] H.G. Sharma, A. Prakash, Flow of a second order fluid in a curved pipe, Indian J. Pure Appl. Math. 8 (1977) 546-557.

[18] Y. Fan, R.I. Tanner, N. Phan-Thien, Fully developed viscous and viscoelastic flows in curved pipes, J. Fluid Mech. 440 (2001) 327-357.

[19] L. Helin, L. Thais, G. Mompean, Numerical simulation of viscoelastic Dean vortices in a curved duct, J. Non-Newton. Fluid Mech. 156 (2009) 84-94.

[20] M. Boutabaa, L. Helin, G. Mompean, L. Thais, Numerical study of Dean vortices in developing Newtonian and viscoelastic flows through a curved duct of square cross-section, C.R. Mec. 337 (2009) 40-47.

[21] P.A.J. Mees, K. Nandakumar, J.H. Masliyah, Instabilities and transitions of flow in a curved square duct: the development of two pairs of dean vortices, J. Fluid Mech. 314 (1996) 227-246.

[22] D.M. Chilcott, J.M. Rallison, Creeping flow of dilute polymer solutions past cylinders and spheres, J. Non-Newton. Fluid Mech. 29 (1988) 381-432.

[23] R.B. Bird, C.F. Curtiss, R.C. Armstrong, O. Hassager, Dynamics of Polymeric Liquids, Kinetic Theory, vol. 2, John Wiley \& Sons, New York, 1987.

[24] D.F. James, Boger fluids, Annu. Rev. Fluid Mech. 41 (2009) 129-142.

[25] P.J. Oliveira, F.T. Pinho, G.A. Pinto, Numerical simulation of on-linear elastic flows with a general collocated finite-volume method, J. Non-Newton. Fluid Mech. 79 (1998) 1-43

[26] M.A. Alves, P.J. Oliveira, F.T. Pinho, A convergent and universally bounded interpolation scheme for the treatment of advection, Int. J. Numer. Methods Fluids 41 (2003) 47-75.

[27] P.J. Oliveira, Method for time-dependent simulations of viscoelastic flows: vortex shedding behind cylinder, J. Non-Newton. Fluid Mech. 101 (2001) 113137.

[28] K.H. Winters, A bifurcation study of laminar flow in a curved tube of rectangular cross-section, J. Fluid Mech. 180 (1987) 343-369. 